\title{
Exploring Reionization-era Quasars. III. Discovery of 16 Quasars at $6.4 \lesssim z \lesssim 6.9$ with DESI Legacy Imaging Surveys and the UKIRT Hemisphere Survey and Quasar Luminosity Function at $z \sim 6.7$
}

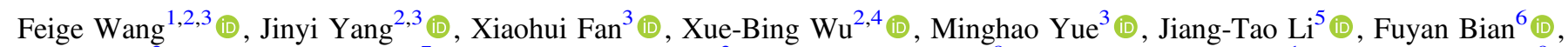

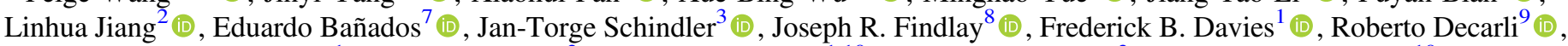

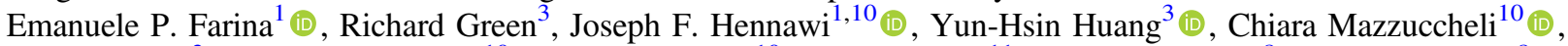

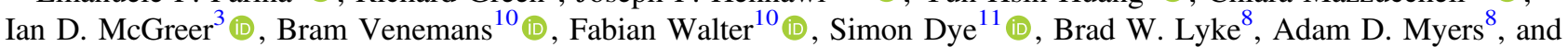
Evan Haze Nunez ${ }^{12,13}$ (1)

${ }^{1}$ Department of Physics, University of California, Santa Barbara, CA 93106-9530, USA; fgwang@physics.ucsb.edu

${ }^{2}$ Kavli Institute for Astronomy and Astrophysics, Peking University, Beijing 100871, People's Republic of China ${ }^{3}$ Steward Observatory, University of Arizona, 933 North Cherry Avenue, Tucson, AZ 85721, USA

${ }^{4}$ Department of Astronomy, School of Physics, Peking University, Beijing 100871, People's Republic of China

${ }^{5}$ Department of Astronomy, University of Michigan, 311 West Hall, 1085 S. University Ave, Ann Arbor, MI, 48109-1107, USA

${ }^{6}$ European Southern Observatory, Alonso de Córdova 3107, Casilla 19001, Vitacura, Santiago 19, Chile

${ }^{7}$ The Observatories of the Carnegie Institution for Science, 813 Santa Barbara Street, Pasadena, CA 91101, USA

${ }^{8}$ University of Wyoming, Physics \& Astronomy 1000 E. University, Dept. 3905, Laramie, WY 82071, USA

${ }^{9}$ INAF-Osservatorio di Astrofisica e Scienza dello Spazio, via Gobetti 93/3, I-40129, Bologna, Italy

${ }^{10}$ Max Planck Institut für Astronomie, Königstuhl 17, D-69117, Heidelberg, Germany

${ }^{11}$ School of Physics and Astronomy, Nottingham University, University Park, Nottingham, NG7 2RD, UK

${ }^{12}$ Department of Physics and Astronomy, California State Polytechnic University, 3801 West Temple Ave., Pomona, CA 91768, USA

${ }^{13}$ Department of Physics and Astronomy, El Camino College, 16007 Crenshaw Blvd., Torrance, CA 90506, USA Received 2018 October 28; revised 2019 April 25; accepted 2019 June 20; published 2019 October 8

\begin{abstract}
This is the third paper in a series aimed at finding reionization-era quasars with the combination of DESI Legacy imaging Surveys (DELS), the Pan-STARRS1 (PS1) Survey, and near-infrared imaging surveys, such as the UKIRT Hemisphere Survey (UHS), as well as the Wide-field Infrared Survey Explorer (WISE) mid-infrared survey. In this paper, we describe the updated quasar candidate selection procedure, report the discovery of 16 quasars at $6.4 \lesssim$ $z \lesssim 6.9$ from an area of $\sim 13,020 \mathrm{deg}^{2}$, and present the quasar luminosity function (QLF) at $z \sim 6.7$. The measured QLF follows $\Phi\left(L_{1450}\right) \propto L_{1450}^{-2.35}$ in the magnitude range $-27.6<M_{1450}<-25.5$. We determine the quasar comoving spatial density at $\langle z\rangle=6.7$ and $M_{1450}<-26.0$ to be $0.39 \pm 0.11 \mathrm{Gpc}^{-3}$ and find the exponential density evolution parameter to be $k=-0.78 \pm 0.18$ from $z \sim 6$ to $z \sim 6.7$, corresponding to a rapid decline by a factor of $\sim 6$ per unit redshift toward earlier epochs. This indicates that the rapid decline of quasar spatial density at $z>5$ that was found by previous works continues to $z>6$, at a rate significantly faster than the average decline rate between $z \sim 3$ and 5 . We measured quasar comoving emissivity at $z \sim 6.7$, which indicates that high-redshift quasars are highly unlikely to make a significant contribution to hydrogen reionization. The broad absorption line quasar fraction at $z \gtrsim 6.5$ is measured to be $\gtrsim 22 \%$. In addition, we also report the discovery of six additional quasars at $z \sim 6$ in the Appendix.
\end{abstract}

Key words: early universe - galaxies: active - galaxies: high-redshift - quasars: general

\section{Introduction}

Absorption spectra of $z>6$ quasars reveal complete GunnPeterson (GP) absorption troughs, indicating a rapid increase in the intergalactic medium (IGM) neutral fraction toward higher redshift, marking the end of the reionization epoch at $z \sim 6$ (see Fan et al. 2006 and references therein). However, the GP trough saturates even with a small neutral hydrogen fraction of $x_{\mathrm{HI}} \gtrsim 10^{-4}$ and becomes insensitive to higher $\mathrm{HI}$ densities. If the IGM is mostly neutral, there would be appreciable absorption redward of the wavelength of the Ly $\alpha$ emission line due to the sum of the Rayleigh scattering and would give rise to longwavelength off-resonance absorptions in the form of a damping wing profile (e.g., Miralda-Escudé 1998; Madau \& Rees 2000).

Despite many efforts made in the past decade, there are only three quasars currently known at $z>7$ (Mortlock et al. 2011; Bañados et al. 2018; Wang et al. 2018) and $~ 20$ quasars at $z \gtrsim 6.5$ (e.g., Venemans et al. 2013, 2015; Matsuoka et al. 2016, 2018b, 2018a; Mazzucchelli et al. 2017; Reed et al. 2017; Wang et al. 2017) discovered to date. This is caused by the combination of a rapid decline of quasar spatial number density toward high redshifts (e.g., Fan et al. 2001; McGreer et al. 2013; Jiang et al. 2016; Yang et al. 2016) and the lack of deep near-infrared (NIR) surveys over a large (i.e., $\gtrsim 10,000 \mathrm{deg}^{2}$ ) sky area.

Currently, the damping wing analyses have only been performed in the line of sight of two known $z>7$ quasars (Bolton et al. 2011; Mortlock et al. 2011; Bosman \& Becker 2015; Greig et al. 2017; Bañados et al. 2018; Davies et al. 2018a), which limits our current knowledge of the reionization history. In spite of that, combining those constraints from quasar absorption spectra with results from the most recent constraints on the declining $\operatorname{Ly} \alpha$ visibility (e.g., Pentericci et al. 2014) and the abundance of highredshift galaxies (e.g., Beckwith et al. 2006; Illingworth et al. 2013) and the cosmic microwave background polarization measurements (Planck Collaboration et al. 2018), current data strongly suggest a peak of reionization activity and emergence of the earliest galaxies and active galactic nuclei (AGNs) at $7<z<11$ (Robertson et al. 2015). This highlights the need to expand the search for quasars at $z \gtrsim 7$. 
Which sources dominate the ionizing photon budget is another key question in understanding the cosmic ionization history (e.g., Madau \& Haardt 2015). Measurement of quasar luminosity function (QLF) at the epoch of reionization (EoR) directly yields the ionizing radiation output from quasars and will help to solve this long-standing question. In addition, the QLF encodes information about the buildup of supermassive black holes (SMBHs) and provides key insights into understanding the BH growth history (e.g., Willott et al. 2010) and the coevolution of SMBHs with their hosts (e.g., Carilli \& Walter 2013; Venemans et al. 2017; Decarli et al. 2018). However, to determine the QLF accurately at high redshift is extremely difficult. Not only does it require a large uniformly selected quasar sample, but the sample needs to be statistically complete.

The QLF at $z \sim 5$ is well measured over a wide luminosity range $\left(-30 \lesssim M_{1450} \lesssim-23\right)$ in the past few years (McGreer et al. 2013; Yang et al. 2016; McGreer et al. 2018), which suggests that the QLF can be described with a double powerlaw function with a very steep bright-end slope of $\beta \sim-3.6$ (Yang et al. 2016), a flatter faint-end slope of $\alpha \sim-2.0$ (McGreer et al. 2013, 2018), and a characteristic magnitude of $M_{1450}^{*} \sim-27$. However, the parameters of the $z \sim 6$ QLF are still debated, Jiang et al. (2016) found that the bright-end slope is $\beta=-2.8$ using the Sloan Digital Sky Survey (SDSS) quasar sample and changes to $\beta=-2.56$ when including fainter Stripe 82 quasars. The faint-end slope and the characteristic magnitude are even less constrained owing to the small number of known faint $z \sim 6$ quasars (e.g.; Willott et al. 2010; Kashikawa et al. 2015; Jiang et al. 2016). More recently, Kulkarni et al. (2019) found that the QLF at $z \sim 6$ has a much steeper bright-end slope $(\alpha=-5.50)$, a very bright characteristic magnitude $\left(M_{1450}^{*}=-29.2\right)$, and a faint-end slope of $\beta=-2.4$, while Matsuoka et al. (2018c) found that the QLF at $z \sim 6$ has a bright-end slope of $\alpha=-2.73$ and a much flatter faint-end slope with $\beta=-1.23$. Nevertheless, these results provide one common conclusion: the contribution of quasars to hydrogen reionization at $z \sim 5-6$ is subdominant.

By combining the QLF measurements at different redshifts, one can investigate the cosmic evolution of quasar activities. Fan et al. (2001) found that the quasar density exponentially declines from $z \sim 3$ to $z \sim 6$ with an exponential density evolution parameter of $k=-0.47$. McGreer et al. (2013) found that the decline rate of quasar number density is more pronounced to $z \sim 6$ with $k=-0.7$, which is confirmed by Jiang et al. (2016) with a larger quasar sample at $z \sim 6$. These studies further indicate that the contribution of ionizing photons to hydrogen reionization from luminous quasars at $z \sim 6$ is minimum.

Measuring the QLF at higher redshift is more challenging, due to the combination of the rapid decline of quasar number density, the large number of contaminations, and the need for wide and deep NIR surveys. Venemans et al. (2013) attempted to estimate the QLF at $z>6.5$ using three $z>6.5$ quasars over $\mathrm{a} \sim 300 \mathrm{deg}^{2}$ area from the VISTA Kilo-degree Infrared Galaxy Survey (VIKING; Arnaboldi et al. 2007) and found that it is consistent with the number density of quasars at $z \sim 6$ with an exponential density evolution parameter of $k=-0.47$. However, their early results have large uncertainties due to the small number of quasars and limited sky coverage. Thus, a large statistically complete and uniformly selected quasar sample at $z>6.5$ is needed to measure the QLF at the EoR.
In Wang et al. (2017, hereafter Paper I), we demonstrate that the combination of the Dark Energy Spectroscopic Instrument $(D E S I)^{14}$ Legacy Imaging Surveys (DELS; Dey et al. 2019), NIR surveys like the UKIRT Hemisphere Survey (UHS; Dye et al. 2018), and the Wide-field Infrared Survey Explorer (WISE; Wright et al. 2010) mid-infrared survey enable us to search for very high redshift quasars over a large sky coverage. We present the discovery of a $z=6.63$ quasar in Paper I and a luminous $z=7.02$ quasar in Wang et al. (2018, hereafter Paper II).

In this paper, we present the updated quasar selection procedure by further including data from the Pan-STARRS1 (PS1) Survey (Chambers et al. 2016). We report the discovery of 16 quasars at $6.4 \lesssim z \lesssim 6.9$ and five quasars at $z \sim 6$ and present the QLF at $z \sim 6$ 6.7. The paper is organized as follows: In Section 2 we briefly introduce imaging surveys and present our updated quasar selection procedure. In Section 3 we describe our follow-up spectroscopic observations for quasar candidates. In Section 4 we report our new discoveries, along with measurements of individual quasar properties and statistical properties of the entire sample. In Section 5 we present the selection completeness and derive the $z \sim 6.7$ QLF measurement. In Section 6 we discuss the quasar spatial density evolution and the contribution of quasars to the cosmic hydrogen reionization. We summarize our results in Section 7. Finally, we report the discovery of six additional $z \sim 6$ quasars in the Appendix.

Following our previous papers, optical magnitudes are reported on the $\mathrm{AB}$ system with Galactic extinction (Schlegel et al. 1998) corrected, and NIR and mid-infrared magnitudes are reported on the Vega system. We adopt a standard $\Lambda \mathrm{CDM}$ cosmology with Hubble constant $H_{0}=70 \mathrm{~km} \mathrm{~s}^{-1} \mathrm{Mpc}^{-1}$ and density parameters $\Omega_{\mathrm{M}}=0.3$ and $\Omega_{\Lambda}=0.7$.

\section{Quasar Candidate Selection}

At $z \sim 7$, the $\operatorname{Ly} \alpha$ emission line in the quasar spectrum redshifts to $\sim 1.0 \mu \mathrm{m}$. Thus, quasars at $z \sim 7$ are characterized by their very red $z-J$ color owing to the presence of neutral hydrogen at high redshift that absorbs most of the emission blueward of the Ly $\alpha$ emission line in quasar spectra. As a result, we need both deep optical and NIR photometry to select quasars at $z \sim 7$.

\subsection{Imaging Data}

For optical bands, we mainly used data from DELS, ${ }^{15}$ which consists of three different imaging surveys: the Dark Energy Camera Legacy Survey (DECaLS), the Beijing-Arizona Sky Survey (BASS; Zou et al. 2018), and the Mayall z-band Legacy Survey (MzLS). These three surveys jointly image $\sim 14,000 \mathrm{deg}^{2}$ of the extragalactic sky visible from the Northern Hemisphere in three optical bands $(g, r$, and $z)$. The DECaLS survey covers $\sim 9000 \mathrm{deg}^{2}$ of the extragalactic sky with decl. $\leqslant 32^{\circ}$, and the BASS +MzLS surveys cover $\sim 5000 \mathrm{deg}^{2}$ of sky with decl. $\geqslant 32^{\circ}$. There is a total region of $\sim 300 \mathrm{deg}^{2}$ at $32^{\circ} .5<$ decl. $<34.5$, where DECaLS overlaps with BASS+MzLS. An overview of DELS surveys can be found in Dey et al. (2019). We also include PS1 photometric data in our selection, which provides a $3 \pi$ sky coverage in $g_{p s 1}, r_{p s 1}, i_{p s 1}, z_{p s 1}$, and $y_{p s 1}$ bands. The PS1 photometric data were obtained from MAST Casjobs PS1

\footnotetext{
14 http://desi.lbl.gov/

15 http://legacysurvey.org/
} 
Table 1

Photometric Information of Data Sets Used in This Paper

\begin{tabular}{lccccc}
\hline \hline Survey & Band & Depth $(5 \sigma)$ & Sky Area $\left(\mathrm{deg}^{2}\right)$ & AB Offset & References \\
\hline DELS (DR4+DR5) & $g, r, z$ & $24.0,23.5,22.5$ & 13,020 & $\ldots$ & Dey et al. (2019) \\
PS1 & $g_{p s 1}, r_{p s 1}, i_{p s 1}, z_{p s 1}, y_{p s 1}$ & $23.3,23.2,23.1,22.3,21.3$ & 30,940 & $\ldots$ & Chambers et al. (2016) \\
UHS & $J$ & 20.5 & 12,700 & 0.938 & Dye et al. (2018) \\
UKIDSS & $J$ & 20.5 & 5,200 & 0.938 & Lawrence et al. (2007) \\
VHS & $J$ & 21.1 & 20,000 & 0.937 & McMahon et al. (2013) \\
VIKING & $J$ & 22.1 & 1350 & 0.937 & Arnaboldi et al. (2007) \\
ALLWISE & $W 1$ & $20.3^{\mathrm{b}}$ & 41,253 & 2.699 & Wright et al. (2010) \\
\hline
\end{tabular}

Notes. All magnitudes in this table are in the AB system. The conversion factors from VEGA to AB are listed in the fifth column.

${ }^{a}$ The depth for DELS is for those areas with only one photometric pass.

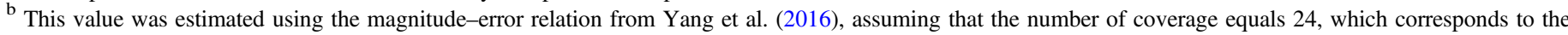
mean coverage of the ALLWISE data set.

Archive. ${ }^{16}$ Although the PS1 survey is shallower than DELS, it has an additional $y_{p s 1}$ band, which is redder and narrower than the reddest $z$ band of DELS. At NIR, we combine UHS DR1 with public data from UKIRT Infrared Deep Sky Survey (UKIDSS; Lawrence et al. 2007) DR10, VISTA Hemisphere Survey (VHS; McMahon et al. 2013) DR5, and VIKING DR4. The UHS and UKIDSS data were obtained from the WFCAM Science Archive, ${ }^{17}$ while the VHS data and VIKING data were obtained from the VISTA Science Archive. ${ }^{18}$ In addition, we used the ALLWISE release ${ }^{19}$ of the WISE data, which combines the original WISE survey (Wright et al. 2010) with data from the NEOWISE (Mainzer et al. 2011) post-cryogenic phase. Because UHS DR1 only provides $J$-band photometry, we only used $J$ band in our selection in order to have a homogeneous selection procedure over the whole DELS footprint. The basic characteristics of these imaging surveys are listed in Table 1.

\section{2. z $\sim 7$ Quasar Candidate Selection}

We started from DELS data release 4 (DR4, MzLS+BASS data) and data release 5 (DR5, DECaLS data), which contain $\sim 0.2$ billion and $\sim 0.7$ billion sources, respectively. The photometric selection procedure of high-redshift quasar candidates in our survey consists of the following seven steps: (1) We first select targets that have $z$-band detection but are not observed/not detected (at $5 \sigma$ level) in $g$ and/or $r$ bands. This results in a total of $\sim 0.3$ billion sources. (2) Then we cross-matched this catalog with the PS1 data release 1 (DR1) catalog using a 2". 0 search radius and removed those targets detected in PS1 $g_{p s 1}, r_{p s 1}$, and $i_{p s 1}$ bands at $5 \sigma$ levels. (3) We then selected targets that have both DELS $z$ and PS1 $y_{p s 1}$ bands with at least $7 \sigma$ detections, have PS1 $y_{p s 1}$ band brighter than $21.5 \mathrm{mag}$ and fainter than $15 \mathrm{mag}$, and have PS1 $z_{p s 1}$ band undetected in $5 \sigma$ or with PS1 $z_{p s 1}-y_{p s 1}>1.5$. (4) We performed forced photometry on PS1 images using an aperture radius of 1 "! 0 for all sources with ALLWISE counterparts within 2 ". 0 separation and removed those with $i_{p s 1 \text {,forced }}$ brighter than 23.1 or $z_{p s 1 \text {,forced }}-y_{p s 1 \text {,forced }}<1.7$. (5) We further rejected targets identified as extended sources in both PS1 photometry (magnitude differences between aperture photometry and point-spread function [PSF] photometry larger than 0.3) and DELS photometry (type not equal to "PSF"). Considering that the astrometry uncertainties in both PS1 and DELS are relative small (i.e., $\lesssim 0$ "' 2 ), we further

\footnotetext{
16 http://mastweb.stsci.edu/ps1casjobs/

$17 \mathrm{http}: / /$ wsa.roe.ac.uk/index.html

18 http://horus.roe.ac.uk/vsa/

19 http://wise2.ipac.caltech.edu/docs/release/allwise/
}

rejected targets with distance between PS1 and DELS positions larger than 1"'0. (6) We cross-matched our candidates with infrared photometric catalogs from UHS, UKIDSS, VHS, and VIKING and rejected those targets with $y_{p s 1}-J>2.0$ or $J-W 1<1.5$ if they are detected in $J$ band. Figure 1 shows the $y_{p s 1}-J / J-W 1$ color-color diagram of high-redshift quasars and Galactic cool dwarfs, as well as our color cuts. (7) We finally visually inspected those candidates. Objects visible in any of PS1 $g_{p s 1}, r_{p s 1}, i_{p s 1}$ and DELS $g, r$ bands, affected by cosmic rays, or contaminated by nearby bright stars are removed.

The selection procedure described above yields a total of 121 quasar candidates left for spectroscopically follow-up observations. We refer to these dropouts as our main quasar candidates in the following sections. Our criteria for targeting $z \sim 7$ quasar candidates are summarized as follows:

$$
\begin{gathered}
\mathrm{S} / \mathrm{N}\left(g, r, g_{p s 1}, r_{p s 1}, i_{p s 1}\right)<5.0 \\
\mathrm{~S} / \mathrm{N}\left(z, y_{p s 1}\right)>7.0 \\
z>16,15<y_{p s 1}<21.5 \\
\mathrm{~S} / \mathrm{N}\left(z_{p s 1}\right)<5.0 \text { or } z_{p s 1}-y_{p s 1}>1.5 \\
i_{p s 1, \text { forced }}>23.1, z_{p s 1, \text { forced }}-y_{p s 1, \text { forced }}>1.7 \\
y_{p s 1}-J<2.0 \\
J-W 1>1.5 .
\end{gathered}
$$

\subsection{Supplementary Quasar Selection}

Recently, large-area surveys for high-redshift quasars have identified a number of high-luminosity quasars with $z>5$ (Wang et al. 2015, 2016; Wu et al. 2015). These quasars are detected in some of the dropout bands owing to their extreme brightness. Our selection procedure presented in Section 2.2 requires nondetections in bands bluer than $z$. On the other hand, strong gravitationally lensed quasars would also be missed by requiring nondetections in bluer bands, as the lensing galaxy would contribute flux in those bands (e.g., McGreer et al. 2010). In order to recover such a quasar population at $z \gtrsim 6.5$ and also expand our quasar-searching sky area, we started from the PS1 DR1 catalog and selected targets with very red colors $\left(\left(g_{p s 1}, \quad r_{p s 1}, \quad i_{p s 1}\right)-y_{p s 1}>3.0, z_{p s 1}-y_{p s 1}>1.5\right) \quad$ at Galactic latitude greater than $5^{\circ}$. Then we cross-matched this catalog with all available NIR photometry data (UHS, VHS, VIKING, 2MASS) and the ALLWISE photometry catalog. We then used the $y_{p s 1}-J / J-W 1$ color-color diagram (Figure 1) and $W 1-W 2>0.4$ (Wang et al. 2016) to reject Galactic cool 


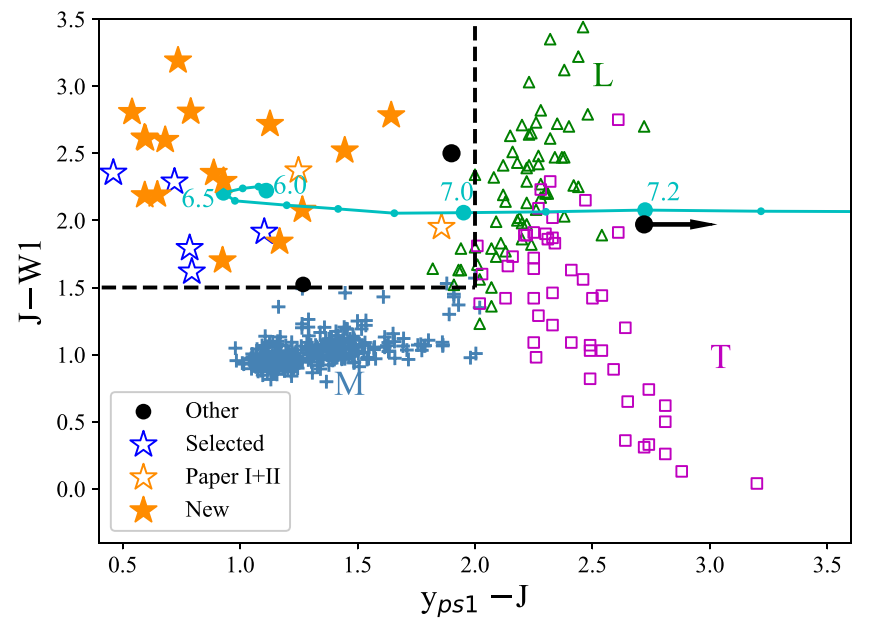

Figure 1. The $y_{p s 1, A B}-J_{\mathrm{VEGA}}$ vs. $J_{\mathrm{VEGA}}-W 1_{\mathrm{VEGA}}$ color-color diagram. The cyan line and cyan filled circles represent the color-redshift relation predicted using simulated quasars (McGreer et al. 2013; Yang et al. 2016) from $z=6.0$ to $z=7.3$, in steps of $\Delta z=0.1$. The large cyan circles highlight the colors at $z=6.0,6.5,7.0$, and 7.2. The orange open stars denote two $z>6.5$ quasars reported in Papers I and II and, and orange filled stars denote $z \gtrsim 6.4$ quasars found in this paper. The open blue stars depict previously known $z>6.5$ quasars that were recovered by our selections. The small black circle denotes a previously known $z>6.5$ quasar that had all PS1, NIR, and WISE detections. The two larger black circles present two known $z>7$ quasars (Mortlock et al. 2011; Bañados et al. 2018) with $y_{p s 1}$ from forced photometry on PS1 images. The steel blue crosses, green open triangle, and magenta open squares depict the positions of M, L, and T dwarfs, respectively (Kirkpatrick et al. 2011; Best et al. 2015).

dwarfs. In the work reported here, we only observed 11 highpriority candidates in this supplementary selection for spectroscopic observations.

\section{Spectroscopic Observations}

We obtained spectroscopic follow-up observations of the quasar candidates with the MMT/Red Channel spectrograph (Schmidt et al. 1989), MMT/MMIRS (McLeod et al. 2012), MMT/Binospec (Fabricant et al. 1998), Magellan/FIRE (Simcoe et al. 2008), Magellan/LDSS3-C (Stevenson et al. 2016), KECK/ DEIMOS (Faber et al. 2003), LBT/MODS (Byard \& O'Brien 2000), Gemini/GMOS (Hook et al. 2004) and P200/DBSP (Oke \& Gunn 1982), over 25 observing nights from 2016 April to 2018 July.

We observed 33 candidates with the MMT $6.5 \mathrm{~m}$ telescope, with 26 main candidates targeted with the Red Channel Spectrograph, 5 main candidates targeted with the MMIRS spectrograph, and 1 main candidate and 1 supplementary candidate observed with Binospec. We used the 270 line $\mathrm{mm}^{-1}$ grating on the Red Channel spectrograph centered at $9000 \AA$, providing wavelength coverage from 7200 to $10800 \AA$. We used the 1 ." 0 or 1 ." 5 slits depends on seeing conditions, providing resolutions of $R \sim 640$ and $R \sim 510$, respectively. MMIRS is a wide-field NIR imager and multiobject spectrograph. We used the HK grism with the $z J$ filter, which provides a wavelength coverage from $9500 \AA$ to $1.3 \mu \mathrm{m}$ and a resolution of $R \sim 1000$ with a 1 !" $^{\prime} 0$ slit. Binospec is a new imaging spectrograph with dual $8^{\prime} \times 15^{\prime}$ fields of view. We used 270 line $\mathrm{mm}^{-1}$ grating centered at $7400 \AA$ with a $1{ }^{\prime \prime} 0$ slit, which provides a resolution of $R \sim 1340$ and wavelength coverage from 6130 to $10120 \AA$.

We observed 27 main candidates and 7 supplementary candidates with Magellan/FIRE. FIRE is an IR echelle/long-slit spectrograph on the $6.5 \mathrm{~m}$ Magellan/Baade Telescope. In order to improve the efficiency, we used the high throughput mode, which provides a resolution of $R \sim 300-500$ from $K$ band to $J$ band. The typical exposure time for each target is 5-10 minutes. We further observed those FIRE-confirmed high-redshift quasars with MMT/Red Channel, LBT/MODS, KECK/DEIMOS, and Gemini/GMOS-N to get optical spectra.

We observed two main candidates with DBSP on the Palomar 200-inch Hale telescope. We used the G316 grating centered at $7500 \AA$ with a 1 ". 5 slit, which provides a resolution of $R \sim 1000$ at $7500 \AA$. Two main candidates were targeted with LBT/MODS using the red grating and a 1 ". 2 slit, which delivers a resolution of $R \sim 1200$ from $5000 \AA$ to $1 \mu \mathrm{m}$. Two main candidates were observed with Magellan/LDSS3-C using the VPH red grating with 1 !"0 slit, which delivers a resolution of $R \sim 1360$ from $6000 \AA$ to $1 \mu \mathrm{m}$. We observed three supplementary candidates using KECK/DEIMOS with $830 \mathrm{G}$ grating.

In total, we spectroscopically observed 65 main candidates and 11 supplementary candidates. The data obtained from Red Channel, Binospec, MODS, and DBSP were reduced using standard IRAF routines. The GMOS data were reduced using the Gemini IRAF packages. The data obtained with FIRE and MMIRS were reduced using a custom set of Python routines (Wang et al. 2017), which includes dark subtraction, flat-fielding, sky subtraction, wavelength calibration, and flux calibration. The data obtained with DEIMOS were reduced using the XIDL ${ }^{20}$ suite of astronomical routines in the Interactive Data Language, which was developed by X. Prochaska and J. Hennawi.

\section{Results}

\subsection{Discovery of 16 New Quasars at $6.4 \lesssim \mathrm{z} \lesssim 6.9$}

Four spectroscopic observed candidates do not have any signal owing to poor weather conditions. Other spectroscopic observed candidates were identified to be high-redshift quasars, Galactic cool dwarfs, or "nonquasars" without any obvious break and/or emission line, which are the main features of high-redshift quasars. ${ }^{21}$ In Papers I and II, we have reported two quasars from the main sample, DELS J1048-0109 at $z=6.6759$ and DELS J0038-1527 at $z=7.02$. In Fan et al. (2019), we reported one strong gravitationally lensed quasar (UHS J0439+1634) at $z=6.511$ from the supplementary sample, including follow-up Hubble Space Telescope imaging and detailed lens model results. All redshifts measured from (sub)millimeter emission lines from the host galaxy have four digits, redshifts measured from $\mathrm{Mg}$ II broad emission lines have three digits, and redshifts measured from Ly $\alpha$ have two digits throughout the paper.

Here we report an additional 16 new quasars at $6.4 \lesssim$ $z \lesssim 7.0$. Fifteen of them come from our main sample, and one object, J210219.22-145854.0, is from our supplementary sample. J2102-1458 is not covered by our main selection because it is outside the DELS footprint and thus not included in our QLF measurements in the following sections. The details of the spectroscopic observations of these newly discovered quasars are listed in Table 2. Figure 2 shows the discovery spectra of these quasars. For those quasars with spectra available only in the optical, we measured quasar redshifts from Ly $\alpha$ and $\mathrm{N}$ V lines by fitting the observed spectra to the

\footnotetext{
${ }^{20}$ http://www.ucolick.org/ xavier/IDL/

21 The list of nonquasars is available from the corresponding author on reasonable request.
} 
Table 2

Observational Information of 16 New Quasars Reported in This Paper

\begin{tabular}{|c|c|c|c|c|}
\hline Name & Telescope & Instrument & Exposure (s) & OBS-DATE (UT) \\
\hline DELS J041128.63-090749.8 ${ }^{\mathrm{a}}$ & MMT & Red Channel & 2700 & 20171226 \\
\hline DELS J070626.39+292105.5 & MMT & Red Channel & 2700 & 20171226 \\
\hline DELS J080305.42+313834.2 & MMT & Red Channel & 6000 & 20180116,20180120 \\
\hline DELS J082931.97+411740.4 & MMT & Red Channel & 2700 & 20171226 \\
\hline DELS J083737.84+492900.4 & MMT & Red Channel & 3600 & 20170320 \\
\hline DELS J083946.88+390011.5 & MMT & Red Channel & 3600 & 20170321 \\
\hline DELS J091013.63+165629.8 & MMT & Red Channel & 6300 & 20171226,20180120 \\
\hline DELS J091054.53-041406.8 & Magellan/Gemini & FIRE/GMOS-N & $900 / 4800$ & $20180417 / 20180516$ \\
\hline DELS J092347.12+040254.4 ${ }^{\mathrm{b}}$ & MMT & Red Channel & 6000 & 20180120,20180203 \\
\hline DELS J110421.59+213428.8 & Magellan/LBT & FIRE/MODS ${ }^{\mathrm{c}}$ & $300 / 3600 \times 2$ & $20170604 / 20180112$ \\
\hline DELS J113508.93+501133.0 & MMT & Red Channel & 1800 & 20171226 \\
\hline DELS J121627.58+451910.7 & MMT & Red Channel & 2400 & 20180118 \\
\hline DELS J131608.14+102832.8 & Magellan/MMT & FIRE/Red Channel & $500 / 2700$ & $20170604 / 20171226$ \\
\hline DELS J153532.87+194320.1 & Magellan/LBT & FIRE/MODS & $600 / 4500$ & $20170604 / 20180707$ \\
\hline DELS J162911.29+240739.6 ${ }^{\mathrm{d}}$ & Hale 200 inch & DBSP & 15900 & $20160908,20160909,20160911$ \\
\hline VHS J210219.22-145854.0 & Magellan/KECK & FIRE/DEIMOS & $900 / 12000$ & $20170801 / 20170914$ \\
\hline
\end{tabular}

Notes.

a This quasar was discovered by Pons et al. (2019) independently.

b This quasar was discovered by Matsuoka et al. (2018a) independently.

${ }^{\mathrm{c}}$ We used binocular mode by using both MODS1 and MODS2.

${ }^{\mathrm{d}}$ This quasar was discovered by Mazzucchelli et al. (2017) independently.

e This quasar was selected from the supplementary sample relying on its more complete spatial coverage.

SDSS quasar template (Vanden Berk et al. 2001) using a visual recognition assistant for quasar spectra software (ASERA; Yuan et al. 2013). The typical redshift errors are about 0.03 owing to the combination of low spectral resolution and strong absorptions blueward of Ly $\alpha$. We have already obtained NIR spectra for some of these quasars, for which we estimate the redshifts by fitting $\mathrm{Mg}$ II emission lines (J. Yang 2019, in preparation). The redshift uncertainties for quasars that have NIR spectra are usually around 0.01 . Note that the redshift uncertainties quoted here do not take the possible shift compared with (sub)millimeter emission lines from quasar host galaxies, which could be up to several thousand kilometers per second (e.g., Decarli et al. 2018). We listed redshifts and photometric information of newly discovered quasars in Table 3. There are four quasars that do not have any available NIR photometry. For these objects, we obtained additional $J$-band photometry with UKIRT/WFCAM (Casali et al. 2007).

We use the continuum magnitude at rest frame $1450 \AA$ in the determination of the QLF. At $z>6.3$, the rest frame $1450 \AA$ is redshifted to observed wavelengths longer than $1.06 \mu \mathrm{m}$, which is beyond the useful wavelength coverage of our optical spectra. Thus, it is not possible to estimate the $1450 \AA$ magnitudes by directly fitting a power law to the discovery spectra shown in Figure 2. Instead, we scale the composite spectra of luminous low-redshift quasars (Selsing et al. 2016) to the Galacticextinction-corrected $J$-band photometry of each quasar. Then we estimate the $1450 \AA$ magnitudes from the scaled composite spectrum. In Table 3, we list the apparent and absolute AB magnitudes at rest frame $1450 \AA$ in Column (3) $\left(m_{1450}\right)$ and Column (4) $\left(M_{1450}\right)$, respectively.

Figure 3 shows the redshift and $M_{1450}$ distribution of all quasars at $z \geqslant 6.3$ from the literature, as well as our newly discovered quasars. The ongoing Subaru High-z Exploration of Low-Luminosity Quasars (SHELLQs) project (Matsuoka et al. 2016, 2018a, 2018b) is focusing on faint quasars (i.e., $\left.M_{1450} \gtrsim-25.5\right)$. In comparison, we are focusing on the parameter space on the redshift and magnitude plane (i.e., at $z>6.5$ and $M_{1450}<-25.5$ ), where only $\sim 10$ previously known quasars were discovered from multiple quasar surveys (Mortlock et al. 2011; Venemans et al. 2013, 2015; Mazzucchelli et al. 2017; Reed et al. 2017; Tang et al. 2017; Bañados et al. 2018). More importantly, these newly discovered bright $z>6.5$ quasars are crucial for probing the cosmic reionization history by searching for and investigating the damping wing absorption features with future high-quality spectroscopy and more accurate redshift measurements.

\subsection{Notes on Individual Quasars}

DELS J083946.88+390011.5. J0839+3900 is a broad absorption line (BAL) quasar at $z=6.905$ with strong blueshifted N V absorption. We obtained a deep Gemini/GNIRS spectrum, which shows that J0839+3900 is a low-ionization BAL (LoBAL) quasar with strong blueshifted $\mathrm{Mg}$ II absorptions. It is the highest-redshift LoBAL quasar known. The NIR spectrum and related physical parameter measurements, together with NIR spectra of other $z>6.5$ quasars, will be reported in a subsequent paper (J. Yang 2019 , in preparation).

DELS J092347.12+040254.4. J0923+0402 is a BAL quasar. It was independently discovered by Matsuoka et al. (2018a).

DELS J091054.53-041406.8. J0910-0414 is a BAL quasar. It was initially identified as a $z \sim 6.8$ quasar from our low signal-to-noise ratio $(\mathrm{S} / \mathrm{N})$ FIRE spectrum. However, after obtaining a deep GMOS optical spectrum, we found that it is a $z=6.63 \mathrm{BAL}$ quasar where most of the flux blueward of $\mathrm{N} \mathrm{V}$ is absorbed.

DELS J070626.39+292105.5. J0706+2921 is the most luminous $z>6.5$ quasar known to date (J0439+1634 reported in Fan et al. 2019 is brighter but has lower intrinsic luminosity after correcting for lensing magnification). It has an absolute magnitude of $M_{1450}=-27.51$ and is about 0.3 mag brighter than the previous record holder (Venemans et al. 2015). 

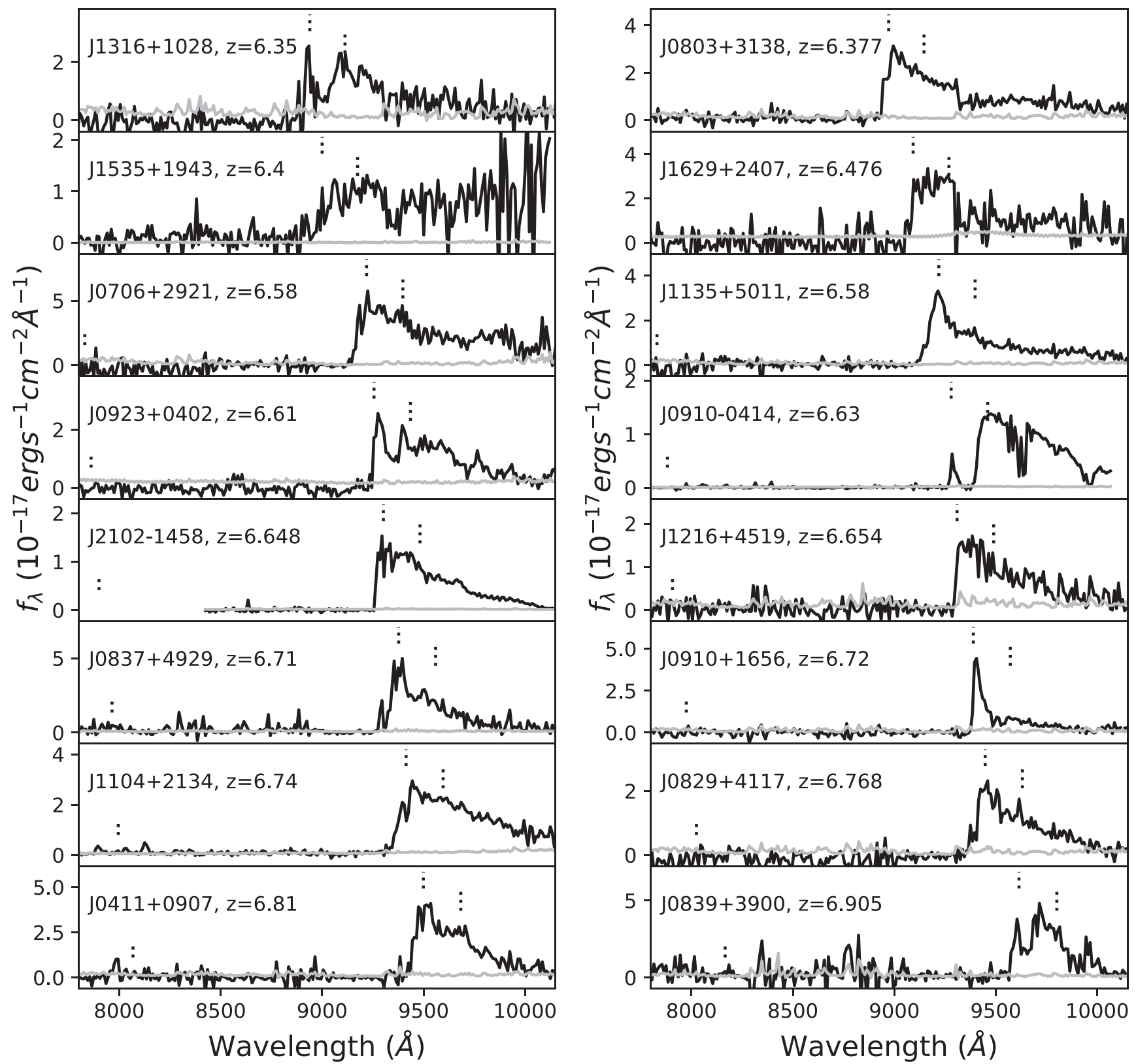

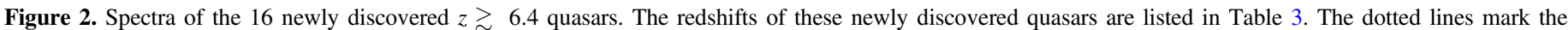

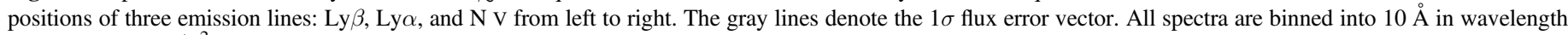
space using the $1 / \sigma^{2}$ weighted mean algorithm.

DELS J162911.29+240739.6. J1629+2407 was independently discovered and reported by Mazzucchelli et al. (2017).

DELS J153532.87+194320.1. J1535+1943 has a very red $y_{p s 1}-J$ color of $\sim 1.7$ compared with other quasars $(\sim 1.0)$ at similar redshifts. The low-S/N spectrum shown in Figure 2 shows that the break blueward of $\operatorname{Ly} \alpha$ is not as sharp as others, which suggests that $\mathrm{J} 1535+1943$ might be a reddened quasar or has a proximate damped Ly $\alpha$ (PDLA) system in front of it.

DELS J131608.14+102832.8. J1316+1028 is a BAL quasar at $z=6.35$ with strong blueshifted $\mathrm{N} \mathrm{V}$ absorption.

\subsection{BAL Quasar Fraction}

BAL quasars show gaseous outflows that cause strong blueshifted absorptions in quasar spectra. Previous studies based on spectral analyses indicated that observed BAL quasars compose about $\sim 15 \%$ of the quasar population at low and intermediate redshifts, without significant redshift dependence (e.g., Hewett \& Foltz 2003; Reichard et al. 2003; Knigge et al. 2008; Gibson et al. 2009). However, Allen et al. (2011) found a strong redshift dependence of the BAL quasar fraction with a factor of $3.5 \pm 0.4$ decrease from $z \sim 4.0$ down to $z \sim 2.0$. The redshift dependence implies that orientation effect alone is not sufficient to explain this trend.

An alternative model that allows cosmic evolution of the BAL quasar fraction is that radiation-driven winds are the likely origin of quasar outflows (e.g., Risaliti \& Elvis 2010). The radiationdriven winds can be generated by the quasar accretion disk under a variety of physical conditions, and the possibility of their existence is a function of physical parameters such as the $\mathrm{BH}$ mass, Eddington ratio, and X-ray-to-UV flux ratio (e.g., Risaliti \& Elvis 2010). Thus, investigating whether the BAL quasar fraction 
Table 3

Properties of 16 New $z \gtrsim 6.4$ Quasars Reported in This Paper

\begin{tabular}{|c|c|c|c|c|c|c|c|c|c|}
\hline Name & Redshift & $m_{1450}$ & $M_{1450}$ & $z_{\mathrm{DELS}, \mathrm{AB}}$ & $y_{\mathrm{ps} 1, \mathrm{AB}}$ & $J_{\mathrm{VEGA}}$ & $W 1_{\text {VEGA }}$ & NIR Survey & QLF \\
\hline DELS J083946.88+390011.5 & $6.905 \pm 0.01^{\mathrm{a}}$ & $20.63 \pm 0.20$ & $-26.29 \pm 0.20$ & $20.92 \pm 0.04$ & $20.24 \pm 0.08$ & $19.45 \pm 0.20$ & $16.64 \pm 0.09$ & UHS & $\mathrm{Y}$ \\
\hline DELS J041128.63-090749.8 & $6.81 \pm 0.03$ & $20.28 \pm 0.12$ & $-26.61 \pm 0.12$ & $20.68 \pm 0.03$ & $20.02 \pm 0.06$ & $19.13 \pm 0.11$ & $16.78 \pm 0.09$ & $\mathrm{New}^{\mathrm{c}}$ & $\mathrm{Y}$ \\
\hline DELS J082931.97+411740.4 & $6.768 \pm 0.006^{\mathrm{a}}$ & $20.52 \pm 0.15$ & $-26.36 \pm 0.15$ & $21.36 \pm 0.04$ & $20.61 \pm 0.11$ & $19.34 \pm 0.15$ & $17.26 \pm 0.14$ & UHS & $\mathrm{Y}$ \\
\hline DELS J110421.59+213428.8 & $6.74 \pm 0.04$ & $20.21 \pm 0.13$ & $-26.67 \pm 0.13$ & $21.06 \pm 0.03$ & $19.94 \pm 0.06$ & $19.01 \pm 0.12$ & $17.31 \pm 0.16$ & UHS & Y \\
\hline DELS J091013.63+165629.8 & $6.72 \pm 0.03$ & $21.30 \pm 0.14$ & $-25.57 \pm 0.14$ & $22.09 \pm 0.13$ & $20.80 \pm 0.15$ & $20.12 \pm 0.13$ & $17.52 \pm 0.20$ & $\mathrm{New}^{\mathrm{c}}$ & $\mathrm{Y}$ \\
\hline DELS J083737.84+492900.4 & $6.710 \pm 0.008^{\mathrm{a}}$ & $20.45 \pm 0.18$ & $-26.42 \pm 0.18$ & $20.66 \pm 0.02$ & $19.86 \pm 0.06$ & $19.27 \pm 0.17$ & $17.08 \pm 0.11$ & UHS & $\mathrm{Y}$ \\
\hline DELS J121627.58+451910.7 & $6.654 \pm 0.01^{\mathrm{a}}$ & $21.27 \pm 0.14$ & $-25.58 \pm 0.14$ & $21.78 \pm 0.12$ & $20.62 \pm 0.09$ & $20.08 \pm 0.13$ & $17.27 \pm 0.13$ & $\mathrm{New}^{\mathrm{c}}$ & $\mathrm{Y}$ \\
\hline VHS J210219.22-145854. $0^{\mathrm{d}}$ & $6.648 \pm 0.01^{\mathrm{a}}$ & $21.36 \pm 0.20$ & $-25.50 \pm 0.20$ & & $20.80 \pm 0.14$ & $20.21 \pm 0.20$ & $17.59 \pm 0.22$ & $\mathrm{New}^{\mathrm{c}}$ & $\mathrm{N}$ \\
\hline DELS J091054.53-041406.8 & $6.63 \pm 0.03$ & $20.49 \pm 0.15$ & $-26.36 \pm 0.15$ & $21.85 \pm 0.14$ & $20.76 \pm 0.13$ & $19.31 \pm 0.14$ & $16.79 \pm 0.10$ & VHS & $\mathrm{Y}$ \\
\hline DELS J092347.12+040254.4 & $6.61 \pm 0.03$ & $20.23 \pm 0.11$ & $-26.61 \pm 0.11$ & $21.18 \pm 0.02$ & $20.20 \pm 0.08$ & $19.08 \pm 0.09$ & $16.36 \pm 0.07$ & LAS & $\mathrm{Y}$ \\
\hline DELS J070626.39+292105.5 & $6.58 \pm 0.03$ & $19.33 \pm 0.08$ & $-27.51 \pm 0.08$ & $20.02 \pm 0.02$ & $19.15 \pm 0.03$ & $18.22 \pm 0.05$ & $15.93 \pm 0.06$ & UHS & $\mathrm{Y}$ \\
\hline DELS J113508.93+501133.0 & $6.58 \pm 0.03$ & $20.65 \pm 0.17$ & $-26.19 \pm 0.17$ & $20.62 \pm 0.04$ & $20.12 \pm 0.07$ & $19.47 \pm 0.16$ & $17.28 \pm 0.12$ & UHS & $\mathrm{Y}$ \\
\hline DELS J162911.29+240739. ${ }^{\mathrm{f}}$ & $6.476 \pm 0.004^{\mathrm{a}}$ & $20.50 \pm 0.18$ & $-26.32 \pm 0.18$ & $20.75 \pm 0.03$ & $20.00 \pm 0.06$ & $19.40 \pm 0.17$ & $16.79 \pm 0.08$ & UHS & $\mathrm{Y}$ \\
\hline DELS J153532.87+194320.1 & $6.4 \pm 0.05$ & $19.79 \pm 0.13$ & $-27.01 \pm 0.13$ & $20.74 \pm 0.04$ & $20.35 \pm 0.06$ & $18.70 \pm 0.11$ & $15.92 \pm 0.05$ & UHS & $\mathrm{N}$ \\
\hline DELS J080305.42+313834.2 & $6.377 \pm 0.006^{\mathrm{a}}$ & $20.28 \pm 0.14$ & $-26.51 \pm 0.14$ & $20.69 \pm 0.10$ & $20.35 \pm 0.10$ & $19.18 \pm 0.12$ & $17.34 \pm 0.16$ & UHS & $\mathrm{N}$ \\
\hline DELS J131608.14+102832.8 & $6.35 \pm 0.04$ & $21.06 \pm 0.17$ & $-25.73 \pm 0.17$ & $21.39 \pm 0.04$ & $20.68 \pm 0.10$ & $19.94 \pm 0.15$ & $16.75 \pm 0.09$ & LAS & $\mathrm{N}$ \\
\hline
\end{tabular}

Notes. The column "NIR Survey" indicates where the $J$-band photometry comes from. The last column indicates whether a quasar is used for QLF measurements.

a The redshift measured from NIR spectra by fitting broad Mg II emission lines (J. Yang et al. 2018, in preparation).

b This quasar was discovered by Pons et al. (2019) independently.

${ }^{c}$ The $J$-band photometry was obtained using UKIRT/WFCam (Programs: U/17B/UA01 and U/17B/D04).

d This quasar was selected from the supplementary sample.

e This quasar was discovered by Matsuoka et al. (2018a) independently.

${ }^{\mathrm{f}}$ This quasar was discovered by Mazzucchelli et al. (2017) independently. 


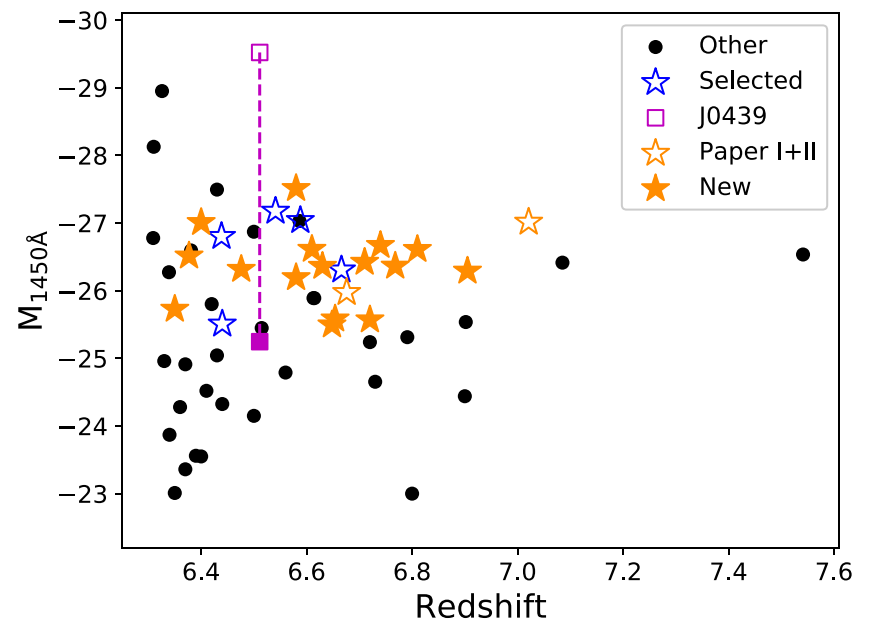

Figure 3. Redshift and absolute magnitude distribution of $z \geqslant 6.3$ quasars. The black circles denote previously known quasars missed by our selection because of their faintness, being outside our searching footprint or at relative low redshift and therefore not satisfying our dropout selection. The two known $z>7$ quasars are also not selected by us because they are undetected by the PS1 survey. The blue open stars represent previously known quasars that satisfy our selection procedures, and the orange open stars denote two quasars published in our Papers I and II. The filled stars denote our newly discovered quasars reported in this paper. The magenta squares denote the most distant gravitationally lensed quasar, J0439+1634 at $z=6.511$, before (open) and after (solid) lensing correction (Fan et al. 2019).

is different at the EoR would help us understand the nature of BAL quasars and probe whether it is only related to the orientation or also affected by other physical parameters.

Four newly discovered quasars in our sample show strong blueshifted $\mathrm{N} V$ absorptions, indicating that they are BAL quasars: $\mathrm{J} 1316+1028$ at $z=6.35, \mathrm{~J} 0923+0402$ at $z=6.61, \mathrm{~J} 0910-0414$ at $z=6.63$, and $\mathrm{J} 0839+3900$ at $z=6.905$. In addition, the NIR spectrum of J0038-1527 shows that it has multiple strong C IV broad absorption troughs (Paper II). We only estimate the BAL fraction in our main quasar sample because it is statistically complete. There are five published PS1 quasars that also satisfy our main selection procedure (See Table 4). Thus, we need to include these five PS1 quasars, J1048-0109 reported in Paper I, and J0038-1527 reported in Paper II but exclude J2102-1458 when counting the BAL fraction. We visually inspected the spectra of five PS1 quasars (Venemans et al. 2015; Mazzucchelli et al. 2017); only PSO J036+03 shows small possible absorption troughs blueward of S IV and C IV emission lines. However, the spectrum of PSO J036+03 in Venemans et al. (2015) shows several unusual bumps, which could be due to flux calibration issues typical of echelle spectra. We treat PSO $\mathrm{J} 036+03$ as a possible BAL quasar. Therefore, the observed BAL quasar faction in our main quasar sample is $5(6) / 23=21.7(26.1) \%$, which is slightly higher than that at lower redshift (e.g., Hewett \& Foltz 2003; Reichard et al. 2003; Knigge et al. 2008; Gibson et al. 2009). Note that the spectra of some quasars do not cover the C IV lines and we do not know whether they are real non-BAL quasars. In addition, the color selection bias would underestimate the BAL quasar fraction by a few percent (Reichard et al. 2003) because BAL quasars usually have slightly redder colors. Thus, the observed BAL quasar fraction given here should be treated as a lower limit. We will revisit this question in detail after collecting NIR spectra for all quasars.

\subsection{Radio Properties}

Bright radio sources at the EoR allow us to study the cosmic reionization by detecting $21 \mathrm{~cm}$ absorptions from intervening neutral IGM (e.g., Carilli et al. 2007; Semelin 2016). In addition, powerful radio jets play a key role in the formation and buildup of SMBHs (e.g., Volonteri et al. 2015). The radioloud fraction of quasars is found to be $\sim 10 \%$ from low redshifts up to $z \sim 6$ (e.g., Jiang et al. 2007; Bañados et al. 2015). However, no radio-loud quasar has been found at $z>6.5$, where the universe is relatively neutral. The large quasar sample presented here allows investigation of the radio properties of early quasars. We cross-matched all known $z \gtrsim 6.5$ quasars presented in this paper and from the literature with the Faint Images of the Radio Sky at Twenty Centimeters (FIRST; Becker et al. 1995) survey and the NRAO VLA Sky Survey (NVSS; Condon et al. 1998). However, none of the known $z>6.5$ quasars are detected in these two radio surveys.

In order to distinguish radio-loud quasars from radio-quiet quasars, many criteria were proposed in the literature (e.g., Kellermann et al. 1989; Stocke et al. 1992). Here we calculate the radio-loudness as $R=f_{6 \mathrm{~cm}} / f_{2500}$ (e.g., Stocke et al. 1992; Jiang et al. 2007), where $f_{6 \mathrm{~cm}}$ and $f_{2500}$ are flux densities at restfame $6 \mathrm{~cm}$ and $2500 \AA$, respectively. We estimate $f_{2500}$ from $m_{1450}$ by assuming $f_{\nu} \propto \nu^{-0.6}$ (Lusso et al. 2015), and we estimate $f_{6 \mathrm{~cm}}$ from the $1.4 \mathrm{GHz}$ observed flux density by assuming $f_{\nu} \propto \nu^{-0.75}$ (e.g., Wang et al. 2007). Figure 4 shows the constraints on radio-loudness of $z>6.5$ quasars with the flux limits of FIRST (1.5 mJy) and NVSS (2.5 mJy). Limited to the depth of FIRST and NVSS surveys, we can only confirm that none of the newly discovered quasars have $R \gtrsim 500$.

In order to further constrain the average radio emission of these quasars, we stacked VLA FIRST images of 17 publicly known $z>6.5$ quasars $(10$ from this work and 7 from the literature) located within the footprint of the FIRST survey. The stacked image reached an rms of $0.037 \mathrm{mJy}$ and is shown in Figure 5. We do not detect any significant radio emission from the stacked image, which limits the mean radio emission of these 17 quasars to be $\lesssim 0.1 \mathrm{mJy}$ at a $3 \sigma$ level. Future deeper radio imaging of these $z>6.5$ quasars is required to identify radio-loud $z>6.5$ quasars and further study their radio emissions.

\section{Quasar Luminosity Function at $z \sim 6.7$}

\subsection{A Complete Quasar Sample}

Since most of our discoveries are from our main sample, we will calculate the QLF only using the main quasar sample. There are 15 quasars reported in this paper from the main sample, 2 quasars reported in Papers I and II, and another 5 quasars discovered by the PS1 high-redshift quasar survey (Venemans et al. 2015; Mazzucchelli et al. 2017) that also satisfy our selection criteria. The previously known $z \gtrsim 6.4$ quasars recovered by our main selection are listed in Table 4. Due to the nondetection requirement in $g, r$, and $i$ bands (Equation (1)) and $z$-dropout cut (Equation (5)) in our selection procedure, our selection is highly incomplete at $z \lesssim 6.4$ (See Section 5.3 for more details). Thus, we further reject three newly discovered quasars (J1535+1943, J0803+3138, and $\mathrm{J} 1316+1028)$ and two additional quasars (P261+19 and P183 $+05)$ at $z<6.45$ from Mazzucchelli et al. (2017) when calculating the QLF. Our main sample missed four $z>6.4$ 
Table 4

Previously Known $z \gtrsim 6.4$ Quasars Recovered by Our Main Selection Procedure

\begin{tabular}{|c|c|c|c|c|c|c|c|c|c|}
\hline Name & Redshift & $m_{1450}$ & $M_{1450}$ & $z_{\text {DELS,AB }}$ & $y_{\mathrm{ps} 1, \mathrm{AB}}$ & $J_{\mathrm{VEGA}}$ & $W 1_{\text {VEGA }}$ & References $^{\mathrm{a}}$ & QLF \\
\hline DELS J003836.10-152723.6 & $7.021 \pm 0.006$ & $19.93 \pm 0.08$ & $-27.01 \pm 0.08$ & $21.65 \pm 0.08$ & $20.61 \pm 0.10$ & $18.75 \pm 0.07$ & $16.80 \pm 0.10$ & (1) & $\mathrm{Y}$ \\
\hline DELS J104819.08-010940.4 & $6.6759 \pm 0.0005$ & $20.89 \pm 0.18$ & $-25.97 \pm 0.18$ & $21.95 \pm 0.05$ & $20.96 \pm 0.14$ & $19.71 \pm 0.17$ & $17.34 \pm 0.17$ & (2) & Y \\
\hline PSO J338.2298+29.5089 & $6.666 \pm 0.0004$ & $20.55 \pm 0.15$ & $-26.31 \pm 0.15$ & $21.05 \pm 0.05$ & $20.22 \pm 0.09$ & $19.43 \pm 0.14$ & $17.81 \pm 0.21$ & (3) & $\mathrm{Y}$ \\
\hline PSO J323.1382+12.2986 & $6.5881 \pm 0.0003$ & $19.80 \pm 0.12$ & $-27.04 \pm 0.12$ & $19.72 \pm 0.02$ & $19.17 \pm 0.02$ & $18.71 \pm 0.11$ & $16.36 \pm 0.07$ & (4) & $\mathrm{Y}$ \\
\hline PSO J036.5078+03.0498 & $6.541 \pm 0.002$ & $19.66 \pm 0.12$ & $-27.18 \pm 0.12$ & $19.98 \pm 0.01$ & $19.30 \pm 0.03$ & $18.52 \pm 0.10$ & $16.73 \pm 0.08$ & (3) & $\mathrm{Y}$ \\
\hline PSO J261.0364+19.0286 & $6.44 \pm 0.05$ & $21.30 \pm 0.19$ & $-25.51 \pm 0.19$ & $21.60 \pm 0.08$ & $20.92 \pm 0.12$ & $20.20 \pm 0.18$ & $17.91 \pm 0.21$ & (4) & $\mathrm{N}$ \\
\hline PSO J183.1124+05.0926 & $6.4386 \pm 0.0004$ & $20.00 \pm 0.10$ & $-26.80 \pm 0.10$ & $20.53 \pm 0.01$ & $19.98 \pm 0.05$ & $18.88 \pm 0.08$ & $16.97 \pm 0.13$ & (4) & $\mathrm{N}$ \\
\hline
\end{tabular}

Note.

${ }^{\mathrm{a}}$ These objects were discovered by several studies: (1) Paper II; (2) Paper I; (3) Venemans et al. (2015); (4) Mazzucchelli et al. (2017). 


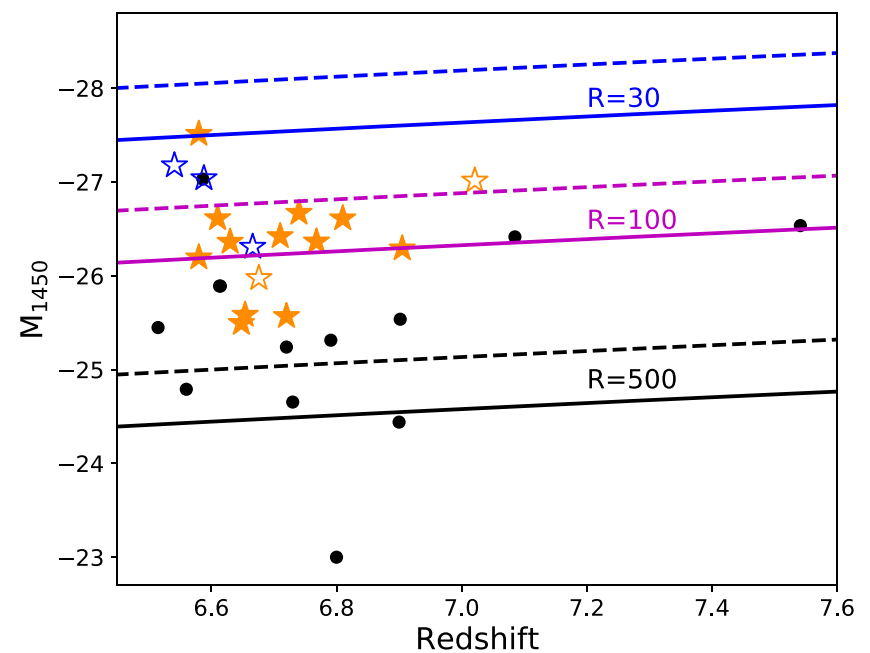

Figure 4. Redshift and absolute magnitude distribution and radio-loudness constraints of $z>6.5$ quasars. The symbols are the same as in Figure 3. The solid lines denote radio-loudness calculated from the FIRST flux limit, and dashed lines denote radio-loudness calculated from the NVSS flux limit. The black, magenta, and blue curves represent $R=500, R=100$, and $R=30$, respectively. It shows that both FIRST and NVSS surveys are too shallow to rule out that those $z>6.5$ quasars are radio-loud quasars with $R \sim 100$. But none of our newly discovered quasars are extremely radio-loud quasars with $R>500$.

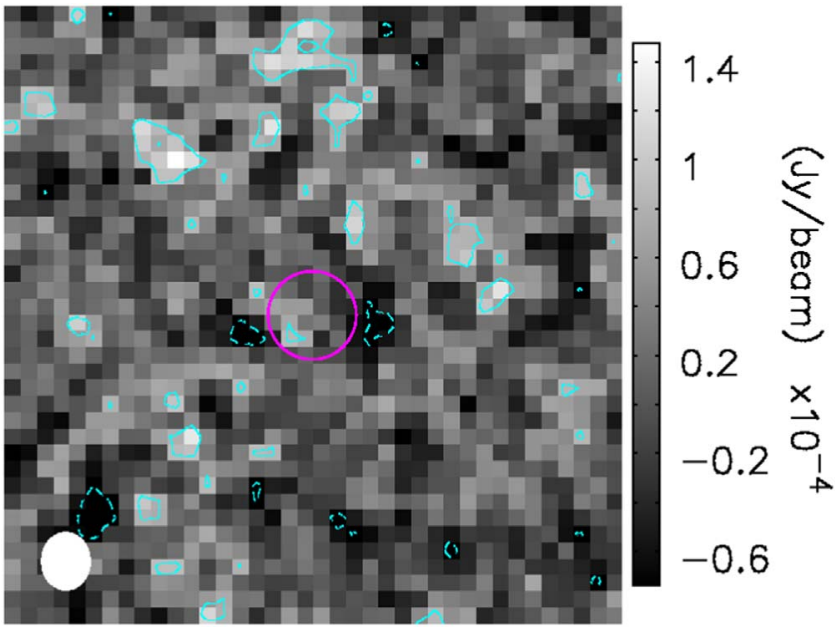

Figure 5. Stacked VLA FIRST image of 17 (10 from this work and 7 from the literature) $z>6.5$ quasars. The white ellipse shows the beam size, and the magenta circle (radius of $5^{\prime \prime}$ ) marks the stacked quasar position. The pixel size of the stacked image is 1 . 8 pixel $^{-1}$. The rms of the stacked image is $\sim 3.7 \times 10^{-2} \mathrm{mJy} \mathrm{beam}^{-1}$, four times better than the FIRST depth. $+2 \sigma$ contours are shown as cyan solid lines, and $-2 \sigma$ contours are shown as cyan dashed lines. No signal is detected at the quasar position.

quasars (P011+09, P167-13, P231-20, and P006+39) discovered by the PS1 quasar surveys (Venemans et al. 2015; Mazzucchelli et al. 2017; Tang et al. 2017) because they do not fall into the DELS footprint.

The final quasar sample we used for the QLF measurement includes 17 quasars with $6.45<z<7.05$ and $-27.6<M_{1450}<$ -25.5. The redshifts of these quasars were measured from Ly $\alpha$ and $\mathrm{Mg}$ II broad emission lines or from [C II] emission lines in submillimeter (Decarli et al. 2018) with a median redshift of $\langle z\rangle \sim 6.7$. The apparent and absolute $\mathrm{AB}$ magnitudes of continuum at rest frame $1450 \AA\left(m_{1450}\right.$ and $\left.M_{1450}\right)$ for those recovered known quasars are derived using the same method as described in Section 4.1.

\subsection{Area Coverage}

Since the PS1 covers the whole DELS footprint and we do not reject any sky area that is not covered by the NIR surveys (i.e., a small area at decl. $>60^{\circ}$ ), the total searching area is basically the footprint of DELS DR4 and DR5. We require quasar candidates to have at least one DELS $z$-band observation. But we do not limit our selections with $g$ - and/ or $r$-band observations; thus, the $g$ - and $r$-band observations do not affect our sky coverage estimate. The DELS DR4 covers $3267 \mathrm{deg}^{2}$ in $z$ band, ${ }^{22}$ and the DELS DR5 covers $9972 \mathrm{deg}^{2}$ in $z$ band. $^{23}$ However, there are $\sim 200 \mathrm{deg}^{2}$ overlap regions observed; thus, we need to avoid double-counting the overlap regions when estimating the area coverage. Instead of adding the DR4 and DR5 coverage, we generate a photometric catalog including both DELS DR4 and DR5 photometric data and only keep one object if it has duplicated detections in DR4 and DR5. We then use the Hierarchical Equal Area isoLatitude Pixelization (HEALPix; Górski et al. 2005) to estimate the sky coverage of DR4+DR5 following Jiang et al. (2016). The final estimated sky area by HEALPix is $13,020 \mathrm{deg}^{2}$, which is consistent with the area estimated by adding DR4 and DR5 coverage and removing overlap regions.

\subsection{Selection Function}

Following our previous works (McGreer et al. 2013, 2018; Jiang et al. 2016; Yang et al. 2016), we use simulations to estimate the completeness of our selection procedure, including the color cuts and flux limits that we applied in Section 2.2. The simulation is performed under the assumption that the shape of quasar spectral energy distributions (SEDs) does not evolve with redshift. We generate a grid of model quasars using the simulations by Yang et al. (2016), which is an updated version of the simulations by McGreer et al. (2013). The modeled quasar spectra are designed to match the colors of $\sim 60,000$ SDSS BOSS quasars in the redshift range of $2.2<z<3.5$ (Ross et al. 2012). Each quasar spectrum consists of a broken power-law continuum, a series of emission lines with Gaussian profiles, and Fe emission templates (Boroson \& Green 1992; Vestergaard \& Wilkes 2001; Tsuzuki et al. 2006). The distributions of spectral features, such as the continuum spectral slope, line equivalent width, and line FWHM, are matched to those of BOSS quasars.

The simulated spectra also involve absorptions from neutral hydrogen absorption in the Ly $\alpha$ forest. Finally, photometry is derived from simulated quasars, and photometric errors are added for each survey by matching the observed magnitude and error relations with a large representative point-source sample (Yang et al. 2016). The PS1 coverage depends on the sky position, and the depth is not uniform. Chambers et al. (2016) give the all-sky distribution of magnitude limits for $50 \%$ and 98\% completeness on the PS1 $3 \pi$ stacked data, which indicates that the PS1 DR1 catalog is $\sim 50 \%$ complete for $y_{p s 1} \gtrsim 21.5$ objects and is $\gtrsim 98 \%$ complete for $y_{p s 1}<21$ objects (except for some low Galactic latitude regions). We need to consider the sky-position-dependent photometric uncertainties caused by

\footnotetext{
22 http://legacysurvey.org/dr4/description/

23 http://legacysurvey.org/dr5/description/
} 


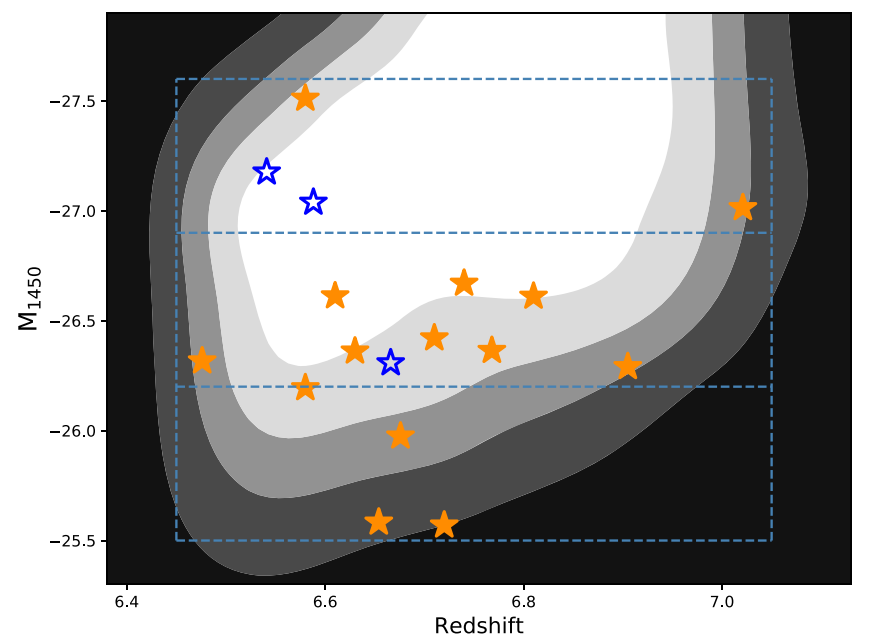

Figure 6. Selection function of our $z \sim 7$ quasar survey. The probability is the fraction of simulated quasars selected by our selection criteria among all simulated quasars in each $\left(M_{1450}, z\right)$ bin. The orange filled stars denote newly discovered quasars reported in this work, and blue open stars are previously known $z>6.45$ quasars. The contours are selection probabilities from 0.7 to 0.1 with an interval of 0.2 .

the PS1 inhomogeneous coverage. To correct this effect in our simulations, we mapped the PS1 spatial surveying depth within our searching area and fit a 2D magnitude-coverage-error relation following the procedure explored by Yang et al. (2016). Similarly, we also apply the same method for the simulated DELS photometry. We refer to McGreer et al. (2013) and Yang et al. (2016) for more detailed descriptions of the simulation.

We use the simulation described above to estimate the completeness of our selection criteria. To derive a selection function, we construct a grid of simulated quasars distributed evenly in $\left(M_{1450}, z\right)$ space with 100 quasars bin $^{-1}$ of $\Delta M_{1450}=$ 0.1 and $\Delta z=0.05$. Then we compute the average selection probability, $p\left(M_{1450}, z\right)$, in each $\left(M_{1450}, z\right)$ bin. The computed selection function in the $\left(M_{1450}, z\right)$ space and the 17 quasars we used for QLF measurement are shown in Figure 6. We note that there are two faint quasars that have probabilities below $30 \%$ : $\mathrm{J} 0910+1656$ at $z=6.72$ and $\mathrm{J} 1216+4519$ at $z=6.654$. In particular, J0910+1656 has a probability of only $\sim 10 \%$. The reason we can select $\mathrm{J} 0910+1656$ is that this quasar has a strong Ly $\alpha$ emission and thus is bright in both $y_{\mathrm{ps} 1}$ and $z_{\mathrm{DELS}}$, although it is faint in rest frame $1450 \AA$. J1216+4519 is not that extreme but has similar situation to $\mathrm{J} 0910+1656$. $\mathrm{J} 1216+4519$ is bright in $y_{\mathrm{ps} 1}$ and $z_{\text {DELS }}$ but faint in $M_{1450}$. There are no quasars found in the highly complete region at redshift between 6.6 and 6.9, which is probably because there is no such bright quasar in our searching area at this redshift range. Not surprisingly, this is because the number density of such luminous quasars at $z>6.6$ is very low. For example, there are only three $z>6.5$ quasars with $M_{1450}$ brighter than -27 previously known at $z>6.5$, with two of them being recovered by our selection and the other one being out of our searching sky area (Mazzucchelli et al. 2017).

Our quasars span a magnitude range of $M_{1450}$ from -27.51 to -25.51 . In order to include a statistical quasar sample in each magnitude bin, we divide our sample into three magnitude bins with $\Delta M_{1450}=0.7 \mathrm{mag}$ over the magnitude range $-25.5<M_{1450}<-27.6$. Figure 7 shows the selection function as a function of redshift in three different luminosity bins.

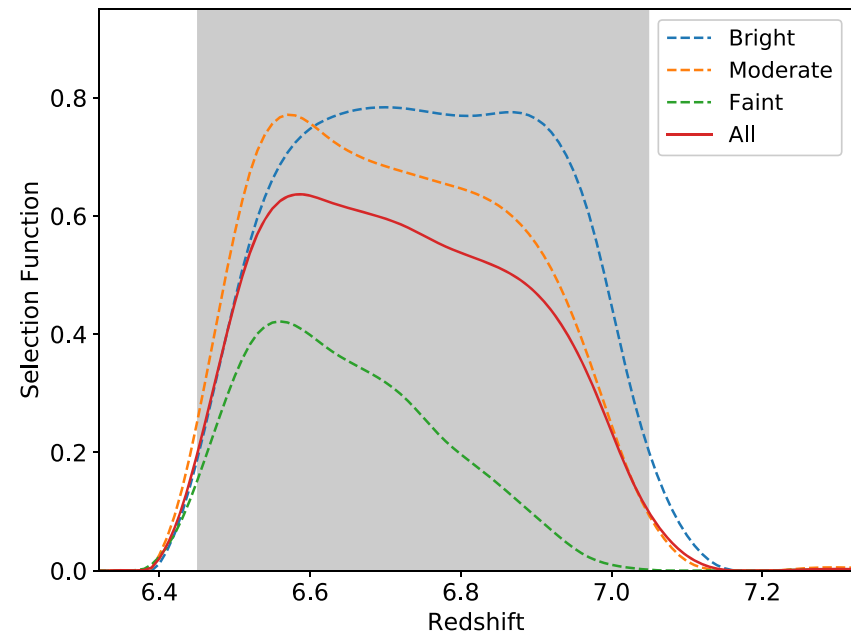

Figure 7. Selection function as a function of redshift for three different luminosity bins. The blue, orange, and green dashed lines denote the selection function for quasars with $-27.6<M_{1450}<-26.9,-26.9<M_{1450}<-26.2$, and $-26.2<M_{1450}<-25.5$, respectively. The red solid line represents the selection function over the whole magnitude range. The shaded region shows the redshift bin we used for calculating QLF.

As expected from the $z$-dropout cut, our main selection procedure has a very sharp change of completeness at $z \sim 6.45$. Because we required strong detections in both PS1 $y_{p s 1}$ and DELS $z$ band, our main selection limits the quasar redshift to be lower than 7.05 and can only select very luminous quasars at $z \gtrsim 6.8$. For these reasons, we choose the redshift range from 6.45 to 7.05 (the shaded region in Figure 7) when calculating the QLF. As shown in Figure 6, the main selection procedure misses very bright quasars at $z \lesssim 6.6$ owing to requirements of nondetections in PS1 $i$ band.

\subsection{Spectroscopic Completeness}

As mentioned in Section 3, we spectroscopically observed 65 main candidates. These 65 observed targets include quasars J1048-0109 and J0038-1527, which have been published in Papers I and II. There are four candidates in our spectroscopically observed sample that can be neither rejected nor confirmed as high-redshift quasars based on available spectra, and we cannot count these four candidates as spectroscopically observed targets. Thus, the overall success rate of our main selection is $29.5 \%(18 / 61)$. The success rate at $y_{p s 1}<20.5$ is very high $(10 / 12=83.3 \%)$ and declines rapidly toward fainter objects because the dropout bands are not deep enough for fainter candidates. There are five more previously known quasars, J0226+0302, J1212+0505, J1724+1901， J2132 +1217 , and $\mathrm{J} 2232+2930$, that can also be treated as spectroscopically observed targets. Hence, 66 out of 121 main sample candidates were spectroscopically observed in total. The $y_{p s 1}$ magnitude distribution of our observed and unobserved candidates is shown in Figure 8. The number of spectroscopically observed candidates is a function of $y_{p s}$-band magnitude, which is used to correct the incompleteness by assuming that the probability of an unobserved candidate to be a quasar is the same as in the observed sample at a certain magnitude. As shown in Figure 8, we do not identify any highredshift quasar at $y_{p s 1}>21.0$, which means that we cannot correct the quasar fraction for unobserved candidates at this magnitude range. Figure 8 shows that the number of main 


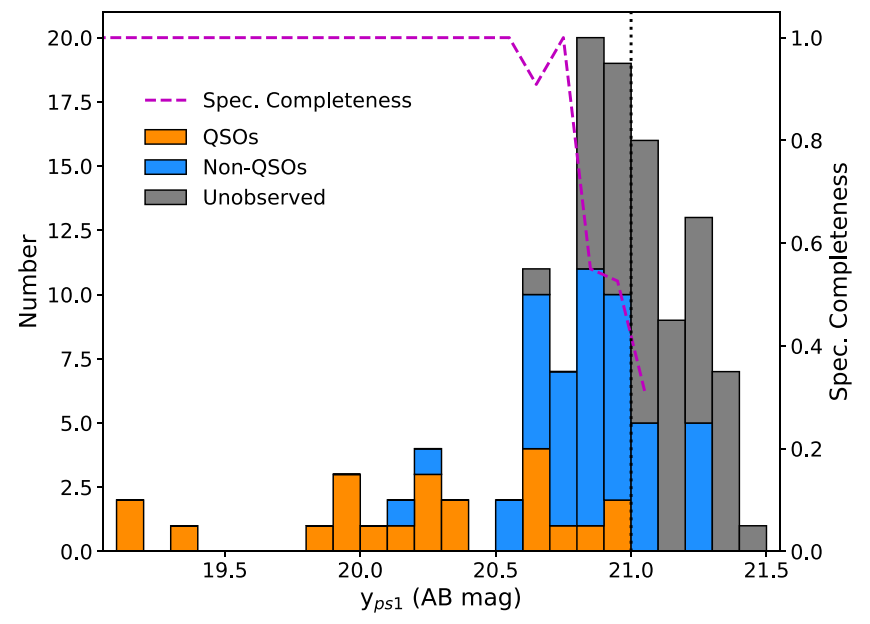

Figure 8. Spectroscopic completeness of our main sample. The magenta dashed line denotes the spectroscopic completeness as a function of $y_{p s 1}$-band magnitude. The histogram is divided into several components filled by different colors and represents newly identified high-redshift quasars (orange), nonquasars (blue), and unobserved candidates (gray). The black dotted line represents $y_{p s 1}=21.0 \mathrm{mag}$, which we treat as our quasar survey flux limit.

candidates drops very fast at $y_{p s 1}>21.0$, which is caused by the fact that the depth of PS1 is not uniform and faint objects can only be detected at the $7 \sigma$ level in deep regions. Considering these two limitations, we only use the $y_{p s 1}<$ 21.0 main sample to calculate the QLF.

\subsection{Luminosity Function at $\mathrm{z} \sim 6.7$}

To compute the binned QLF, we divide our sample into three magnitude bins as mentioned in Section 5.3. Due to the narrow redshift interval of our sample and the small number of highredshift quasars, we only use one redshift bin and do not take into account any redshift evolution within this redshift range. The volume densities of quasars are calculated using the standard $1 / V_{a}$ method (Page \& Carrera 2000), after all incompleteness corrections have been applied for each quasar.

The binned QLF is shown in Figure 9. The QLF can be well characterized by a single power law, $\Phi\left(L_{1450}\right) \propto L_{1450}^{\beta}$, or

$$
\Phi\left(M_{1450}\right)=\Phi^{*} 10^{-0.4(\beta+1)\left(M_{1450}+26\right)},
$$

where we only consider luminosity dependence but ignore redshift evolution over our narrow redshift range. The best fits are $\Phi^{*}=(6.34 \pm 1.73) \times 10^{-10} \mathrm{Mpc}^{-3} \mathrm{mag}^{-1}$ and $\beta=$ $-2.35 \pm 0.22$.

At lower redshifts, QLFs are commonly characterized using a double power law,

$$
\Phi(M, z)=\frac{\Phi^{*}(z)}{10^{0.4(\alpha+1)\left(M-M^{*}\right)}+10^{0.4(\beta+1)\left(M-M^{*}\right)}},
$$

where $\alpha$ and $\beta$ are the faint-end and bright-end slopes, respectively, $M^{*}$ is the characteristic magnitude, and $\Phi^{*}(z)=$ $\Phi^{*}(z=6) \times 10^{k(z-6)}$ is the normalization. Since our binned QLF only covers a narrow luminosity range, we cannot fit $\alpha$ and $M^{*}(z)$. Currently, there is no $z>6.5$ QLF measurement at the faint end, so we fix $\alpha$ and $M^{*}$ to the $z \sim 6 \mathrm{QLF}$ measured by Jiang et al. (2016): $\alpha=-1.90$ and $M^{*}=-25.2$. Here we use least-squares fitting rather than maximum likelihood fitting

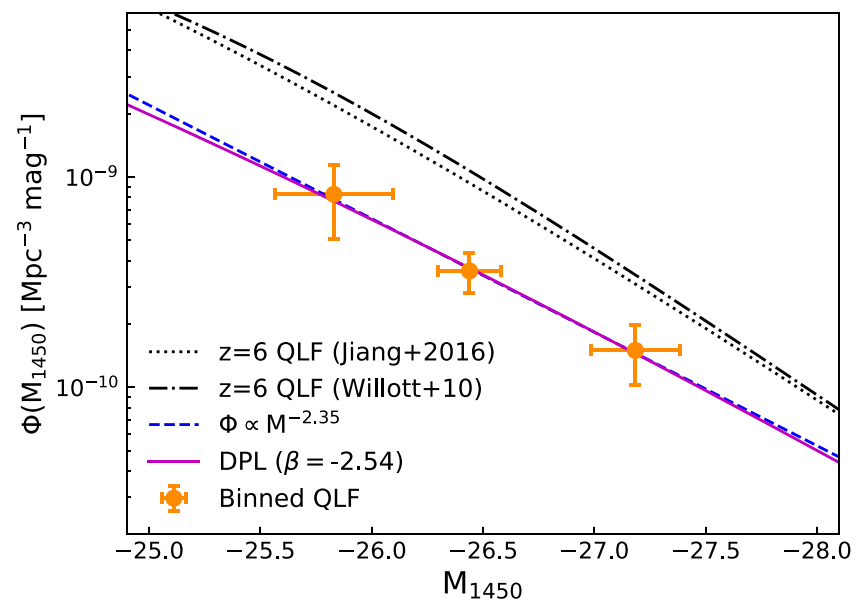

Figure 9. Binned quasar luminosity function. The orange filled circles represent our newly measured binned QLF at $z \sim 6.7$. The blue dashed line is our best fit with $\beta=-2.35 \pm 0.22$. The magenta solid line denotes a double power-law fit. The black dotted and dashed-dotted lines represent $z \sim 6 \mathrm{QLF}$ measured by Jiang et al. (2016) and Willott et al. (2010), respectively. Clearly, the quasar number density at $z \sim 6.7$ is much lower than that at $z \sim 6$.

owing to the lack of faint quasars and unknown faint-end slope and characteristic magnitude. Our least-squares fitting gives $\Phi^{*}=(3.17 \pm 0.85) \times 10^{-9} \mathrm{Mpc}^{-3} \mathrm{mag}^{-1}$ and $\beta=-2.54 \pm$ 0.29 .

At lower redshifts $(z \lesssim 5)$, the QLF has a very steep brightend slope $\beta \leqslant-3$ and a flat faint-end slope $\alpha \sim-2.0$ to -1.5 (e.g., Richards et al. 2006; McGreer et al. 2013; Yang et al. 2016; Schindler et al. 2018). Jiang et al. (2016) measured a slightly flatter bright-end slope of $\beta=-2.8 \pm 0.2$ at $z \sim 6$ with SDSS quasars brighter than $M_{1450}=-25.3$ (see also Willott et al. 2010). However, the bright-end slope changes to $\beta=-2.56 \pm 0.16$ if they fit a single power law to all SDSS quasars $\left(-29.10 \leqslant M_{1450} \leqslant-24.3\right)$. More recently, Kulkarni et al. (2019) measured a much steeper bright-end slope $\beta=-5.05_{-1.18}^{+0.76}$ at $z \sim 6$. The large difference in the brightend slope between Kulkarni et al. (2019) and previous works is mainly because Kulkarni et al. (2019) measured a very bright characteristic magnitude, which is $M^{*}=-29.21$. In other words,the QLF measured by Kulkarni et al. (2019) follows a single power law with a slope of $-2.41_{-0.08}^{+0.10}$ at $M_{1450} \gtrsim-29.0$, which is similar to the single power-law fitting at $-29.10 \leqslant$ $M_{1450} \leqslant-24.3$ by Jiang et al. (2016). We measured the single power-law QLF slope to be $\beta=-2.35 \pm 0.22$ at $z=6.7$ using a sample of quasars with $-27.6<M_{1450}<-25.5$. The slope changes to $\beta=-2.54 \pm 0.29$ if we fix the faint-end slope and characteristic magnitude to be the values derived by Jiang et al. (2016); it changes to $\beta=-2.34 \pm 0.22$ if we fix the bright-end slope and characteristic magnitude to be the values derived by Kulkarni et al. (2019). Our result suggests that the QLF slope does not evolve strongly from $z \sim 6$ (Jiang et al. 2016; Kulkarni et al. 2019) to $z=6.7$ over a magnitude range of $-27.6<M_{1450}<-25.5$.

\section{Discussion}

\subsection{Density Evolution of High-redshift Luminous Quasars}

A rapid decline in the comoving number density of luminous quasars at high redshift was suggested by Fan et al. (2001), who fit an exponential decline to the quasar spatial density, $\rho$ 


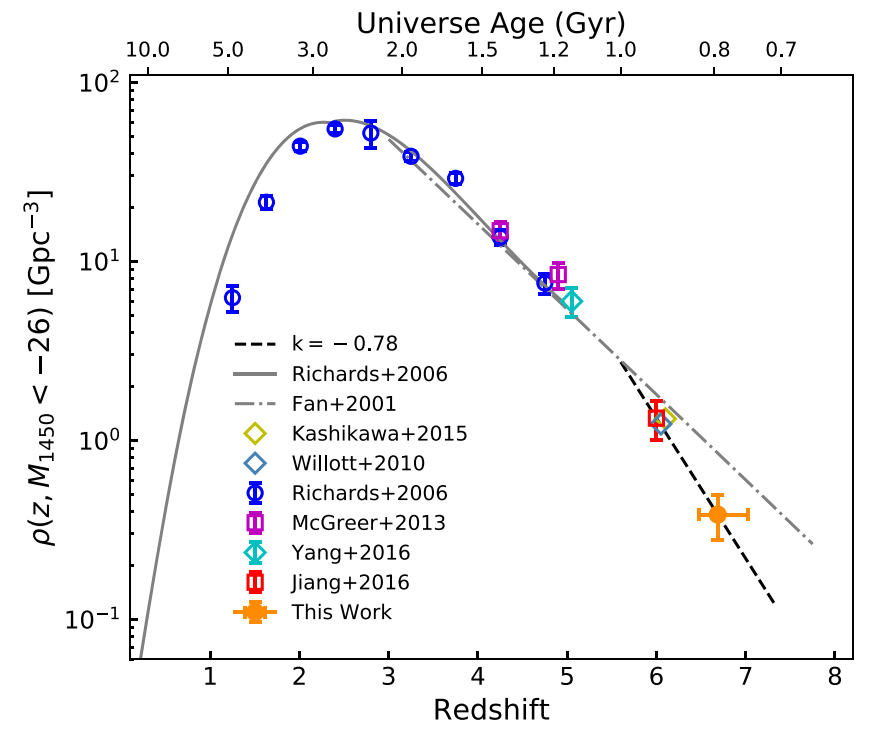

Figure 10. Density evolution of luminous quasars. The gray solid line and dashed line denote the evolution model from Richards et al. (2006) and Fan et al. (2001), respectively. The black dashed line denotes the density evolution model from $z \sim 6$ to $z \sim 6.7$ with $k=-0.78$. The gray solid line and gray dotted-dashed lines are from Richards et al. (2006) and Fan et al. (2001), respectively. The orange filled circle denotes our measurement at the highest redshift. The blue open circles are density measured from the binned SDSS quasar luminosity function (Richards et al. 2006). The magenta and red open squares are densities measured using the binned luminosity function from McGreer et al. (2013) and Jiang et al. (2016), respectively. The cyan, steel-blue, and yellow open diamonds denote densities integrated from the QLF measured by Yang et al. (2016), Willott et al. (2010), and Kashikawa et al. (2015), respectively.

$(<M, z) \propto 10^{k z}$, and found that the density evolves from $z \sim 3$ to $z \sim 6$ with $k=-0.47$. This value has been frequently used in many previous works (e.g., Willott et al. 2010; Kashikawa et al. 2015). An even more rapid decline in the comoving number density from $z \sim 5$ to $z \sim 6(k \sim-0.7)$ is claimed by recent studies (McGreer et al. 2013; Jiang et al. 2016). Here we explore in detail the spatial density evolution of luminous quasars at higher redshifts. The spatial density of quasars brighter than a given magnitude $M$ can be calculated by integrating the QLF:

$$
\rho(<M, z)=\int_{-\infty}^{M} \Phi(M, z) d M .
$$

We can also estimate the density using the $1 / V_{a}$ method based on individual quasars and selection function

$$
V_{a}=\int_{\Delta z} p\left(M_{1450}, z\right) \frac{d V}{d z} d z
$$

where $p\left(M_{1450}, z\right)$ is the selection function at each magnitude and redshift bin. The total spatial density and its uncertainty can then be estimated by

$$
\rho(<M, z)=\sum_{i} \frac{1}{V_{a}^{i}}, \sigma(\rho)=\left[\sum_{i}\left(\frac{1}{V_{a}^{i}}\right)^{2}\right]^{\frac{1}{2}}
$$

We estimate the density of quasars brighter than $M_{1450}<-26$ at $z \sim 6.7$ to be $(0.39 \pm 0.11) \mathrm{Gpc}^{-3} \mathrm{mag}^{-1}$, by summing over all the quasars used for QLF measurement

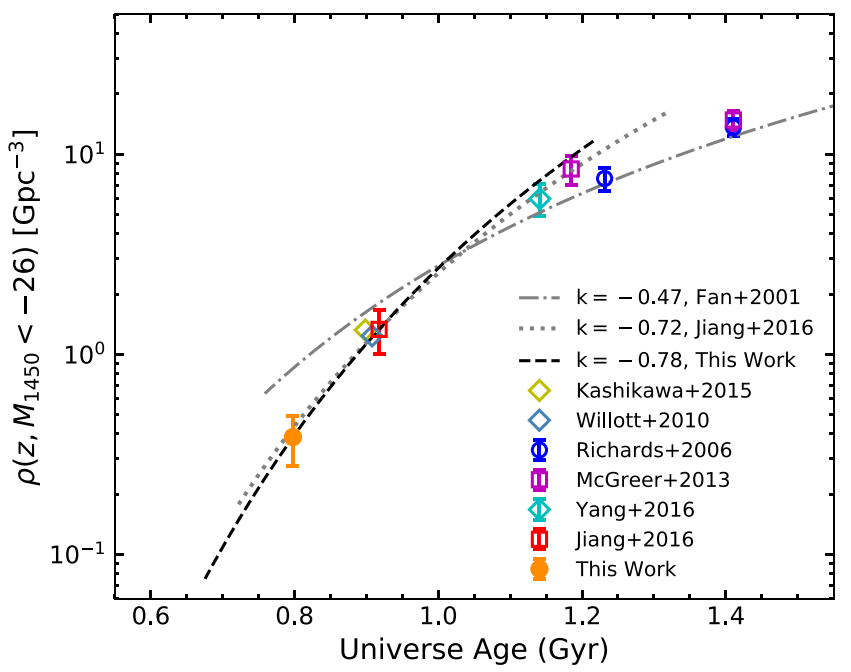

Figure 11. Density evolution of luminous quasars within the first $1.5 \mathrm{Gyr}$ after the big bang. The symbols of different density measurements are the same as in Figure 10. We plotted three different evolution models: $k=-0.47$ from Fan et al. (2001), $k=-0.72$ from Jiang et al. (2016), and $k=-0.78$ from this work.

and with $M_{1450}<-26$ using Equation (12). With the same method, Jiang et al. (2016) measured the density at $z=6$ to be $1.33 \pm 0.33 \mathrm{Gpc}^{-3}$ using a large sample of luminous SDSS quasars. The spatial density of quasars at $z=6.7$ is more than three times lower than that at $z=6$. In Figure 10, we show the estimated quasar spatial density at $z \sim 6.7$, together with the results at $z<5$ from Richards et al. (2006), $z \sim 4-5$ from McGreer et al. (2013), $z \sim 6$ from Jiang et al. (2016), and values derived by integrating the QLFs (e.g., Willott et al. 2010; Kashikawa et al. 2015; Yang et al. 2016) using Equation (10). We derive the exponential density evolution parameter to be $k=-0.78 \pm 0.18$ from $z \sim 6$ to $z \sim 6.7$ by fitting our newly estimated density at $z \sim 6.7$ and the density at $z \sim 6$ from Jiang et al. (2016). Such a steep decline rate indicates that the spatial density of luminous quasars drops by a factor of $\sim 6$ per unit redshift toward earlier cosmic epochs.

McGreer et al. (2013) found that the quasar density declines more rapidly from $z \sim 5$ to $z \sim 6$ than that from $z \sim 3$ to $z \sim 5$ (Fan et al. 2001; Richards et al. 2006), corresponding to $k=$ -0.7 from $z \sim 5$ to $z \sim 6$. This is further confirmed by recent studies by Jiang et al. (2016) and Yang et al. (2019), which found $k=-0.72$ from $z \sim 5$ to $z \sim 6$ and $k=-0.66$ from $z \sim 5$ to $z \sim 5.5$. Figure 11 shows the density evolution of luminous quasars within the first $1.5 \mathrm{Gyr}$ after the big bang, including our new measurements. In this work, we measured $k=-0.78$ from $z=6$ to $z=6.7$, which indicates that the rapid decline of quasar density at $z>5$ continues at $z>6$, i.e., the quasar density from $z \sim 5$ to $z \sim 6.7$ drops about two times faster than that from $z \sim 3$ to 5 , and the decline rate increases from $z>5$ to $z>6$ as shown in Figures 10 and 11. If such a decline extends to higher redshift, we expect to see only one such luminous (i.e., $M_{1450}<-26$ ) quasar over the whole visible sky at $z \sim 9$. This means that we are finally witnessing the first quasars in the EoR, and we will be badly limited by the small number of such quasars when studying the reionization history and SMBH growth history.

Quasar evolution at $z>6$ is limited by the number of $e$ folding times available for $\mathrm{BH}$ accretion. The rapid decline of 
luminous quasar spatial density within such a short cosmic time (i.e., $\sim 121 \mathrm{Myr}$, or three $e$-folding times) indicates that SMBHs could grow rapidly from $z \sim 6.7$ to $z \sim 6$ or they are less radiatively efficient at $z \sim 6.7$. For J0706+2921, the brightest quasar in our sample, it takes $20 e$-folding times, or the age of the universe at $z \sim 6.6$, to grow from a $10 M_{\odot}$ stellar $\mathrm{BH}$, assuming the radiation efficiency $\epsilon=0.1$. The existence of these luminous quasars helps determine whether standard models of radiatively efficient accretion from stellar seeds are still allowed or alternative models of $\mathrm{BH}$ seed formation and BH accretion (super-Eddington or radiatively inefficient) are required (e.g., Volonteri \& Rees 2006).

In addition, the determination of luminous quasar spatial density evolution at high redshift has important consequences in understanding early $\mathrm{BH}$ growth and $\mathrm{BH}$-galaxy coevolution (e.g., Wyithe \& Loeb 2003; Hopkins et al. 2005; Shankar et al. 2010; Mao \& Kim 2016). Combining the dark matter halo mass and duty circle inferred from quasar clustering measurements (Shen et al. 2007), Shankar et al. (2010) predict the QLF at $z>3$ and claim that the rapid drop in the abundance of massive and rare host halos at $z \gtrsim 7$ implies a proportionally rapid decline in the number density of luminous quasars, much stronger than simple extrapolations of the $z=3-6$ luminosity function. Our measurement is consistent with their prediction, with an even stronger declining rate of quasar number density, which requires that these luminous $z>6.5$ quasars reside in even more massive $\left(M_{\text {halo }} \gtrsim 10^{13} M_{\odot}\right)$, less numerous dark matter halos than those of luminous $z \sim 6$ quasars. However, there are currently no conclusive direct observations on whether these high-redshift quasars reside in the most massive dark matter halos. Future deep wide-field imaging and spectroscopy are needed to show whether luminous quasars reside in the most biased environment.

\subsection{Quasar Contribution to Reionization}

Here we estimate the quasar contribution to the ionizing photons at $z \sim 6.7$ based on our newly derived QLF. We first calculate the quasar emissivities by assuming a broken powerlaw quasar SED with an index of $\alpha_{\nu}=-1.7$ at ultraviolet wavelengths, a break at $912 \AA$, and an index of $\alpha_{\nu}=-0.6$ at longer wavelengths (Lusso et al. 2015) and assuming that the escape fraction is $100 \%$. As the characteristic magnitude of QLF at high redshift is highly uncertain, we integrate our QLF down to -18 mag following previous works (Kashikawa et al. 2015; Jiang et al. 2016; Yang et al. 2016), rather than integrating QLF down to $\sim 0.01 M^{*}$ (Madau et al. 1999; Khaire \& Srianand 2015).

As we have measured $\Phi=(6.34 \pm 1.73) \times 10^{-10} \mathrm{Mpc}^{-3}$ $\operatorname{mag}^{-1}$ at $M_{1450}=-26.0$ and the QLF slope to be $\beta=$ $-2.35 \pm 0.22$ at $-27.6<M_{1450}<-25.5$, the ionization photon production rates from quasars at $z=6.7$ are mainly determined by the QLF faint-end slope and the characteristic magnitude. First, we assume that the QLF at $z \sim 6.7$ is a single power law as described by Equation (8). The QLF faint-end slope at $z \sim 6$ was suggested to be $-2.0 \lesssim \alpha \lesssim-1.5$ (e.g., Willott et al. 2010; Kashikawa et al. 2015; Jiang et al. 2016). If the QLF faint-end slope does not evolve from $z \sim 6$ to $z \sim 6.7$, the single power law with $\beta=-2.35$ used here will give a maximum emissivity measurement because our single power-law QLF will overestimate the number of quasars at the faint end. In this case, the double power-law QLF described by

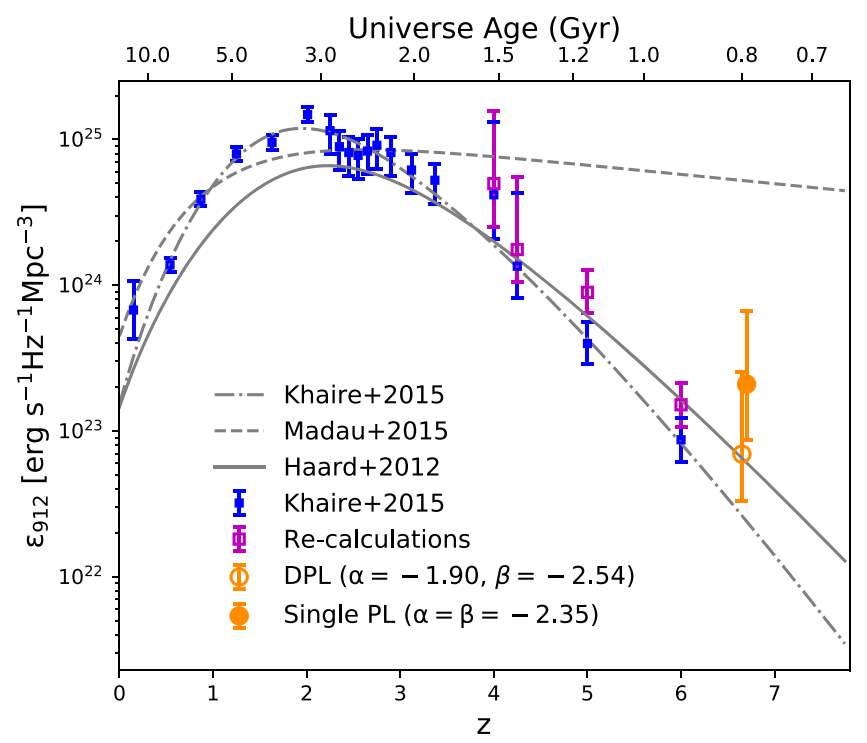

Figure 12. Quasar comoving emissivity at $912 \AA\left(\epsilon_{912}\right)$ vs. redshift $(z)$. The small blue squares are taken from the compilations of Khaire \& Srianand (2015) by integrating QLFs down to $0.01 L_{*}$. The magenta open squares are our recalculations of their $z>4$ points by integrating QLFs down to $-18 \mathrm{mag}$ and assuming a broken power-law quasar SED mentioned in the main text (i.e., same values used for the $z \sim 6.7$ quasar emissivity measurement). The filled and open circles are our calculations at $z \sim 6.7$ by using the single power-law QLF and double power-law QLF, respectively. Note that the open circles are shifted by 0.05 on the $x$-axis for clarity. The solid, dashed-dotted, and dashed lines are models from Haardt \& Madau (2012), Khaire \& Srianand (2015), and Madau \& Haardt (2015), respectively.

Equation (9) with $\alpha$ and characteristic magnitude fixed to the values determined by Jiang et al. (2016) is better suited to estimate quasar comoving emissivity at $z \sim 6.7$. If the break magnitude evolves following the luminosity evolution and density evolution model (McGreer et al. 2013; Yang et al. 2016), the break magnitude at $z \sim 6.7$ will be brighter than -27 , which is also consistent with the recent QLF measurement at $z=6$ (Kulkarni et al. 2019); our best-fit slope is actually the QLF faint-end slope. In this case, the single power law we used here gives the true emissivity measurement. But note that the total quasar comoving emissivity is sensitive to the lower bound of the integral, especially for the single power-law case. Thus, the numbers estimated based on a single power law need to be used with caution.

The quasar comoving emissivity at 1 ryd is estimated to be $\epsilon_{912}=\left(2.10_{-1.23}^{+4.54}\right) \times 10^{23} \mathrm{erg} \mathrm{s}^{-1} \mathrm{~Hz}^{-1} \mathrm{Mpc}^{-3}$ and $\epsilon_{912}=$ $\left(6.95_{-3.64}^{+18.4}\right) \times 10^{22} \mathrm{erg} \mathrm{s}^{-1} \mathrm{~Hz}^{-1} \mathrm{Mpc}^{-3}$, by using the best-fit single power-law QLF $(\beta=-2.35)$ and double power-law QLF $\left(\alpha=-1.90, \beta=-2.54\right.$, and $\left.M^{*}=-25.2\right)$, respectively (Figure 12). To compare with previous works, we also overplot quasar emissivity models (Haardt \& Madau 2012; Khaire \& Srianand 2015; Madau \& Haardt 2015), as well as measurements at lower redshifts, in Figure 12. The integrated emissivity of ionizing photons from quasars at $z \sim 6.7$ is then estimated to be $\dot{\mathcal{N}}_{\text {ion }}=\left(1.86_{-1.09}^{+4.03}\right) \times 10^{49}$ photons $\mathrm{Mpc}^{-3} \mathrm{~s}^{-1}$ and $\dot{\mathcal{N}}_{\text {ion }}=$ $\left(6.17_{-3.23}^{+16.4}\right) \times 10^{48}$ photons $\mathrm{Mpc}^{-3} \mathrm{~s}^{-1}$ for the best-fit single power-law QLF and double power-law QLF, respectively.

The total required photon rate density to balance hydrogen recombination was estimated by Madau et al. (1999), i.e., $\dot{\mathcal{N}}_{\text {ion }}(z)=10^{51.16}\left(\frac{C}{3}\right) \times\left(\frac{1+z}{8}\right)^{3}\left(\frac{\Omega_{b} h_{70}^{2}}{0.08}\right)^{2}$ photons $\mathrm{Mpc}^{-3} \mathrm{~s}^{-1}$, 
where we adopt $\Omega_{b}=0.047$ and $h_{70}=1.0$ in the following calculations. The clumping factor $C$ is suggested to be $\sim 2-3$ at $z \sim 6-7$ by simulations with radiative transfer to capture selfshielding for ionizing backgrounds in the range of those allowed (McQuinn et al. 2011; Kaurov \& Gnedin 2015). For $C=2$, the total required photon rate density at $z=6.7$ is $2.97 \times 10^{50}$ photons $\mathrm{Mpc}^{-3} \mathrm{~s}^{-1}$, and quasars provide $\sim 6.3 \%$ and $\sim 2.1 \%$ of the required photons for the single power-law QLF and double power-law QLF, respectively. For $C=3$, the required photon rate density changes to $4.45 \times$ $10^{50}$ photons $\mathrm{Mpc}^{-3} \mathrm{~s}^{-1}$ and quasars only provide $\sim 4.2 \%$ and $\sim 1.4 \%$ of required photons for the single power-law QLF and double power-law QLF, respectively.

The required photoionization rate can also be inferred directly from measurements of the mean transmitted Ly $\alpha$ flux from quasar spectra (e.g., Meiksin 2005; Becker \& Bolton 2013; D'Aloisio et al. 2018; Davies et al. 2018b). It is related to the total (comoving) emissivity of the sources, $\epsilon_{L}^{S}$, and can be described by $\dot{\mathcal{N}}_{\text {ion }}^{S}=\int_{\nu_{L}}^{\infty} \frac{\epsilon_{L}^{S}}{h_{P}}\left(\frac{\nu}{\nu_{L}}\right)^{-\alpha_{S}} \frac{d \nu}{\nu} \approx \frac{\epsilon_{L}^{S}}{h_{P} \alpha_{s}}=A_{S} \frac{3+\alpha_{\mathrm{MG}}}{3 \alpha_{S}}$ $(1+z)^{\gamma} h$ photons $\mathrm{Mpc}^{-3} \mathrm{~s}^{-1}$, where $h_{\mathrm{P}}$ is the Planck constant, $A_{S}=1.3 \times 10^{52}, \alpha_{\mathrm{MG}}=1$, and $\gamma=-1.6$ (Meiksin 2005). By assuming $\alpha_{s}=1.7$ (Lusso et al. 2015), we measured that quasars only provide $\sim 6.8 \%$ and $\sim 2.3 \%$ of the required photons for the single power-law QLF and double power-law QLF, respectively. The evolution of the comoving production rate of ionizing photons is shown in Figure 13. Although we cannot constrain the faint-end slope and characteristic magnitude of the QLF, the faint-end slope cannot be steeper than the bright-end slope. The ionizing photon production rate estimated by using the single power-law QLF gives either a maximum estimate (if $M^{*}>-25.5$, and -2.35 is the brightend slope) or the true value (if $M^{*}<-27.6$, and -2.35 is the faint-end slope). Based on these results, it is highly unlikely that high-redshift quasars make a significant contribution to hydrogen reionization. As we mentioned before, the total quasar comoving emissivity is very sensitive to the lower bound of the integral for the single power-law case. If there is a significant population of extremely faint AGNs (i.e., $\left.M_{1450} \gtrsim-18\right)$, they would provide a significant contribution to the hydrogen reionization.

\section{Summary}

In this paper, we presented the discovery of 16 quasars at $z \gtrsim 6.4$ and 5 quasars at $z \sim 6$ using modified color selection procedures from Paper I by adding PS1 photometry. Our newly discovered $z \gtrsim 6.4$ quasars span an absolute magnitude range from $\sim-25.5$ to $\sim-27.5$. We have more than doubled the number of known luminous quasars at $z>6.5$ and constructed the first large uniformly selected quasar sample at such a redshift. The statistically uniform $z \gtrsim 6.5$ quasar sample constructed in this paper allows us, for the first time, to estimate the BAL quasar fraction at the EoR, which is $\gtrsim 22 \%$, slightly higher than that at lower redshift. However, future NIR spectroscopy and a larger quasar sample are needed in order to finalize whether BAL quasar fraction evolves with redshift.

After determining the completeness of the selection function estimated using simulated quasars and correcting for spectroscopic incompleteness, we calculated the QLF using a uniform sample of 17 quasars at $6.45<z<7.05$ covering a sky area of $\sim 13,000 \mathrm{deg}^{2}$ to a flux limit of $z_{P S 1}=21$. We measured

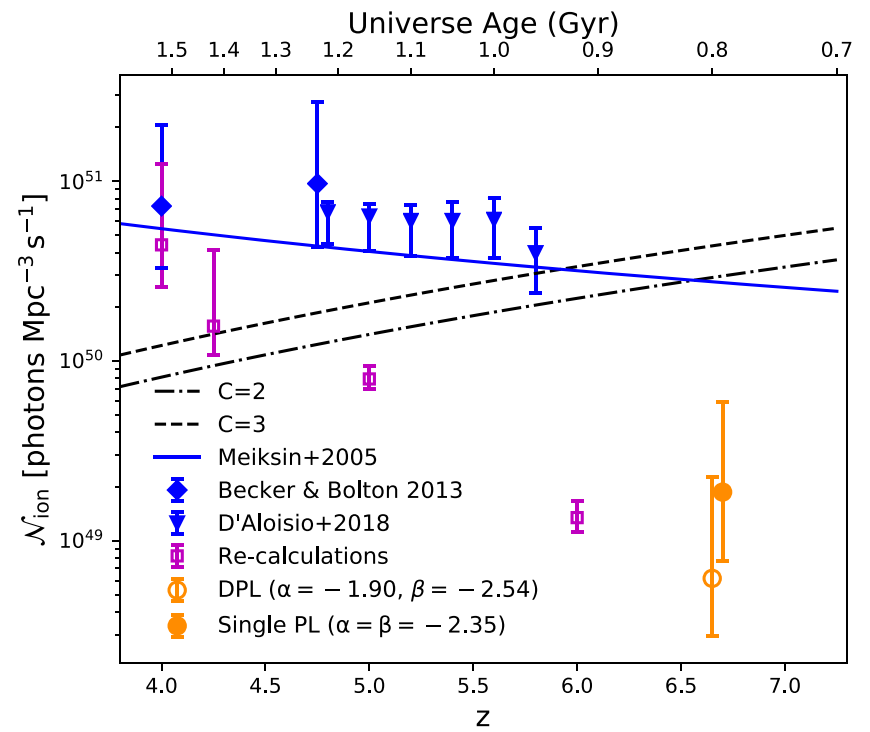

Figure 13. Evolution of the comoving production rate of ionizing photons. The filled and open circles are production rates of ionizing photons at $z \sim 6.7$ based on our single power-law QLF and double power-law QLF, respectively. Note that the open circle was shifted by 0.05 on the $x$-axis for clarity. The magenta open squares are the quasar ionizing photon production rates at lower redshift calculated based on our recalculations of quasar comoving emissivity from Khaire \& Srianand (2015). The blue filled diamonds and triangles denote the required photon production rate inferred from measurements of the mean transmitted Ly $\alpha$ flux by Becker \& Bolton (2013) and D'Aloisio et al. (2018), respectively. The blue solid line represents the required rate inferred from measurements of the mean transmitted Ly $\alpha$ flux from Meiksin (2005). The dashed-dotted and dashed lines are the required photon rate density to balance hydrogen recombination by assuming $C=2$ and $C=3$ (Madau et al. 1999), respectively.

the slope of the QLF to be $\beta=-2.35 \pm 0.22$ within the magnitude range of $-27.6<M_{1450}<-25.5$ by fitting a single power law. If we fit the binned QLF with a double power law and fix the faint-end slope $(\alpha=-1.9)$ and characteristic magnitude $\left(M^{*}=-25.2\right)$, the bright-end slope is measured to be $\beta=-2.54 \pm 0.29$. If we fix the bright-end slope $(\beta=$ $-5.05)$ and characteristic magnitude $\left(M^{*}=-29.21\right)$, the faint-end slope is measured to be $\beta=-2.34 \pm 0.22$. These measurements indicate that the QLF slope does not evolve significantly from $z=6$ to $z=6.7$ within $-27.6<$ $M_{1450}<-25.5$.

We measured the quasar spatial density at $z \sim 6.7$ to be $\rho(<-26.0)=(0.39 \pm 0.11) \mathrm{Gpc}^{-3} \mathrm{mag}^{-1}$ using the $1 / V_{a}$ method. By fitting the spatial density at $z=6$ and $z=6.7$, we find a density evolution parameter of $k=-0.78 \pm 0.18$ from $z \sim 6$ to $z \sim 6.7$, which corresponds to the quasar number density declining by a factor of six per unit redshift toward earlier cosmic epochs. This suggests that the rapid decline rate of quasar density at $z>5$ continues at $z>6$, at a rate significantly faster than the average decline rate between $z \sim 3$ and 5, and the decline rate accelerates from $z>5$ to $z>6$.

We estimated $z \sim 6.7$ quasar comoving emissivity at $1 \mathrm{ryd}$ to be $\epsilon_{912}=\left(2.10_{-1.23}^{+4.54}\right) \times 10^{23} \mathrm{erg} \mathrm{s}^{-1} \mathrm{~Hz}^{-1} \mathrm{Mpc}^{-3}$ and $\epsilon_{912}=$ $\left(6.95_{-3.64}^{+18.4}\right) \times 10^{22} \mathrm{erg} \mathrm{s}^{-1} \mathrm{~Hz}^{-1} \mathrm{Mpc}^{-3}$, using the best-fit single power-law QLF $(\beta=-2.35)$ and double power-law QLF $(\alpha=$ $-1.90, \beta=-2.54)$, respectively. The integrated emissivity of ionizing photons from quasars at $z=6.7$ is estimated to be $\dot{\mathcal{N}}_{\text {ion }}=\left(1.86_{-1.09}^{+4.03}\right) \times 10^{49}$ photons s${ }^{-1} \mathrm{Mpc}^{-3}$ and $\dot{\mathcal{N}}_{\text {ion }}=$ 
$\left(6.17_{-3.23}^{+16.4}\right) \times 10^{48}$ photons $\mathrm{s}^{-1} \mathrm{Mpc}^{-3}$ for the best-fit single power-law QLF and double power-law QLF, respectively. By comparing with the required ionization photon production rate estimated from mean transmitted Ly $\alpha$ flux, our measurements suggest that high-redshift quasars have a very low possibility of being the dominant contributor of hydrogen reionization (i.e., only contribute $<7 \%$ of required ionizing photons).

We are collecting high-quality optical and NIR spectra with large ground-based telescopes. In the forthcoming publications we will give measurements of GP optical depths based on both $\operatorname{Ly} \alpha$ and $\operatorname{Ly} \beta$ forests, search damping wing signatures to constrain the evolution of neutral fraction at the EoR, provide the $\mathrm{BH}$ mass measurements, and explore the $\mathrm{SMBH}$ growth history with the high-quality spectra that we are collecting. We are also observing X-ray emissions from these quasars with Chandra, which will give insights into the accretion status of the earliest SMBHs. This dedicated data set will be an ideal data set for investigating the cosmic reionization history and SMBH formation mechanisms. We are also surveying the [C II] emission line from the quasar host galaxies with ALMA and NOEMA. These observations will provide us excellent opportunities to study the SMBH and host galaxy coevolution in the EoR. Moreover, this unique quasar sample will provide ideal targets for JWST to investigate the environments and host galaxies of these SMBHs.

F.W., J.Y., X.-B.W., and L.J. acknowledge the support by the National Key R\&D Program of China (2016YFA0400703) and the National Science Foundation of China (11533001, 11721303). J.Y., X.F., M.Y., J.-T.S., and I.D.M. acknowledge support from US NSF grant AST-1515115 and NASA ADAP grant NNX17AF28G. J.R.F., B.W.L., A.D.M., and E.H.N. acknowledge support from the National Science Foundation through REU grant AST-1560461.

We acknowledge the use of data obtained at the Gemini Observatory (NOAO program ID: GN-2018A-C-1), which is operated by the Association of Universities for Research in Astronomy (AURA) under a cooperative agreement with the NSF on behalf of the Gemini partnership: the National Science Foundation (United States), the National Research Council (Canada), CONICYT (Chile), the Australian Research Council (Australia), Ministério da Ciência e Tecnologia (Brazil) and Ministerio de Ciencia, and Tecnología e Innovación Productiva (Argentina). We acknowledge the use of the Hale telescope, the Keck I telescope, the LBT telescopes, the Magellan telescopes, the MMT $6.5 \mathrm{~m}$ telescope, and the UKIRT telescope. Observations obtained with the Hale Telescope at Palomar Observatory were obtained as part of an agreement between the National Astronomical Observatories, Chinese Academy of Sciences, and the California Institute of Technology. Observations reported here were obtained at the MMT Observatory, a joint facility of the University of Arizona and the Smithsonian Institution.

We acknowledge the use of BASS, DECaLS, MzLS, PanStarrs, UHS, ULAS, VHS (ESO Programme, 179.A-2010, PI: McMahon), and VIKING (ESO Programme, 179.A-2004, PI: Sutherland) photometric data. This publication makes use of data products from the Wide-field Infrared Survey Explorer, which is a joint project of the University of California, Los Angeles, and the Jet Propulsion Laboratory/California Institute of Technology, and NEOWISE, which is a project of the Jet Propulsion Laboratory/ California Institute of Technology. WISE and NEOWISE are funded by the National Aeronautics and Space Administration.
This research uses data obtained through the Telescope Access Program (TAP), which has been funded by the National Astronomical Observatories, Chinese Academy of Sciences, and the Special Fund for Astronomy from the Ministry of Finance in China.

Facilities: Gemini (GMOS), Hale (DBSP), KECK (DEIMOS), LBT (MODS), Magellan (FIRE, LDSS3-C), MMT (BinoSpec, MMIRS, Red Channel Spectrograph), UKIRT (WFCam).

\section{Appendix \\ Discovery of Six New Quasars at $z \sim 6$}

We keep searching $z \sim 6$ quasars using the traditional $i$-dropout method by combing DELS and infrared surveys mentioned above. The detailed $i$-dropout selection was described in Wang et al. (2017) and will not be repeated here. The only difference is that we used unWISE (Lang 2014; Meisner et al. 2017) photometry instead of ALLWISE photometry when selecting $z \sim 6$ quasar candidates.

For $z \sim 6$ quasar candidates, we first obtained deep $i$-band imaging with the Wide-field Camera mounted on the Wyoming Infrared Observatory (Findlay et al. 2016). After PS1 DR1 was released, we instead used PS1 $i$ band as the dropout band. Following Wang et al. (2017), we took spectroscopic observations for those candidates that satisfy $i-z>2.0$ or $S N(i)<3$. We observed seven $z \sim 6$ quasar candidates that passed our photometric selection with DBSP and Red Channel. The data obtained from Red Channel and DBSP were reduced using standard IRAF routines.

From those observed $i$-dropouts, we identified six new $z \sim 6$ quasars. The observational information of these six quasars is listed in Table 5. Figure 14 shows discovery spectra of these six quasars. The redshifts of these quasars are also measured using ASERA software, with a range of $5.78 \leqslant z \leqslant 6.15$. However, due to poorer quality of these discovery spectra compared with that of $z>6.5$ quasars, the redshift uncertainties are slightly

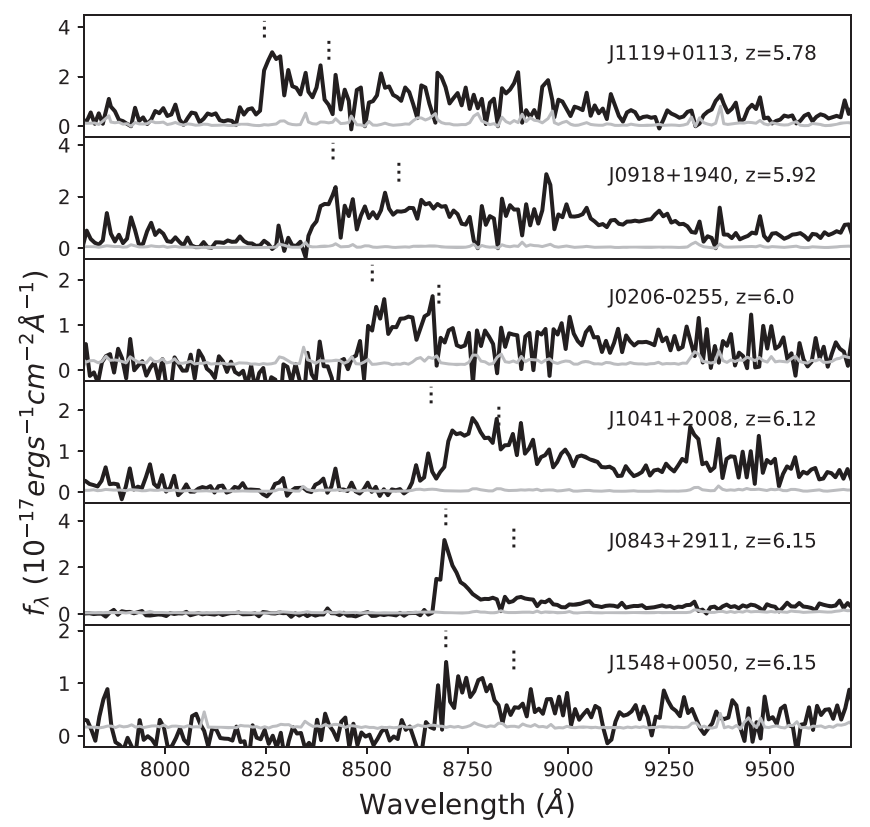

Figure 14. Spectra of six newly discovered $z \sim 6$ quasars. The dotted lines mark the expected positions of $\operatorname{Ly} \alpha$ and $\mathrm{N}$ V emission lines from left to right. The gray lines denote the flux error vector. All spectra are binned into $10 \mathrm{~A}$ in wavelength space using flux error weighted mean. 
Table 5

Observational Information of Six New $z \sim 6$ Quasars Reported in This Paper

\begin{tabular}{lcccc}
\hline \hline Name & Telescope & Instrument & Exposure (s) & Obs-date (UT) \\
\hline DELS J020611.20-025537.8 & Hale 200 inch & DBSP & 3600 & 20160912 \\
DELS J084303.76+291113.4 & Hale 200 inch & DBSP & 1200 & 20170421 \\
DELS J091828.65+194045.0 & MMT & Red Channel & 2400 & 20170331 \\
DELS J104119.15+200824.0 & MMT & Red Channel & 1200 & 20171124 \\
DELS J111921.65+011308.6 & MMT & Red Channel & 2400 & 20170331 \\
DELS J154825.40+005015.5 & Hale 200 inch & DBSP & 5400 & 20170416,20170421 \\
\hline
\end{tabular}

larger. As these $z \sim 6$ quasars are quite faint in NIR $J$ band and the $1450 \AA$ is closer to the PS1 $y_{p s 1}$ band, we scaled the composite spectra to PS1 $y_{p s 1}$-band photometry to measure magnitudes at $1450 \AA$. These quasars have similar luminosities to those of two quasars reported in Wang et al. (2017) and are fainter than the majority of quasars found by the SDSS and PS1 quasar surveys (e.g., Fan et al. 2001; Bañados et al. 2016), but brighter than SHELLQs quasars (e.g., Matsuoka et al. 2016). Table 6 presents the redshift and photometric information of these six newly discovered $z \sim 6$ quasars. 
Table 6

Properties of Six Newly Discovered $z \sim 6$ Quasars

\begin{tabular}{|c|c|c|c|c|c|c|c|c|c|}
\hline Name & Redshift & $m_{1450}$ & $M_{1450}$ & $i_{\mathrm{AB}}$ & $z_{\text {DELS,AB }}$ & $y_{\mathrm{ps} 1, \mathrm{AB}}$ & $J_{\mathrm{VEGA}}$ & $W 1_{\mathrm{VEGA}}{ }^{\mathrm{a}}$ & NIR Survey \\
\hline DELS J154825.40+005015.5 & $6.15 \pm 0.05$ & $21.11 \pm 0.18$ & $-25.62 \pm 0.18$ & $24.58 \pm 0.67^{\mathrm{b}}$ & $20.82 \pm 0.02$ & $21.12 \pm 0.18$ & $19.79 \pm 0.25$ & $17.32 \pm 0.10$ & LAS \\
\hline DELS J084303.76+291113.4 & $6.15 \pm 0.03$ & $20.94 \pm 0.15$ & $-25.79 \pm 0.15$ & $25.09 \pm 2.75^{\mathrm{c}}$ & $20.62 \pm 0.04$ & $20.95 \pm 0.15$ & $20.12 \pm 0.22$ & $17.79 \pm 0.18$ & LAS \\
\hline DELS J020611.20-025537.8 & $6.00 \pm 0.05$ & $20.87 \pm 0.17$ & $-25.82 \pm 0.17$ & $24.08 \pm 1.21^{\mathrm{c}}$ & $21.27 \pm 0.05$ & $20.86 \pm 0.17$ & $20.34 \pm 0.29$ & $18.67 \pm 0.32$ & VHS \\
\hline DELS J104119.15+200824.0 & $6.12 \pm 0.05$ & $20.65 \pm 0.11$ & $-26.08 \pm 0.11$ & $24.02 \pm 0.43^{\mathrm{b}}$ & $20.56 \pm 0.03$ & $20.65 \pm 0.10$ & $19.42 \pm 0.18$ & $17.43 \pm 0.11$ & UHS \\
\hline DELS J091828.65+194045.0 & $5.92 \pm 0.05$ & $20.76 \pm 0.13$ & $-25.91 \pm 0.13$ & $23.32 \pm 0.26^{\mathrm{c}}$ & $20.86 \pm 0.07$ & $20.73 \pm 0.13$ & $19.48 \pm 0.21$ & $16.96 \pm 0.07$ & UHS \\
\hline DELS J111921.65+011308.6 & $5.78 \pm 0.05$ & $21.06 \pm 0.14$ & $-25.57 \pm 0.14$ & $22.75 \pm 0.11^{\mathrm{b}}$ & $20.75 \pm 0.02$ & $20.99 \pm 0.14$ & $19.58 \pm 0.23$ & $16.88 \pm 0.07$ & LAS \\
\hline
\end{tabular}

Notes.

a The WISE W1 magnitudes come from the unWISE photometric catalog

${ }^{\mathrm{b}}$ The $i$-band photometry comes from the PS1 DR1 photometric catalog.

${ }^{\mathrm{c}}$ The $i$-band photometry was obtained with the Wide-field Camera mounted on the Wyoming Infrared Observatory (Findlay et al. 2016).

${ }^{\mathrm{d}}$ This quasar was discovered by Matsuoka et al. (2018b) independently. 


\section{ORCID iDs}

Feige Wang (iD https://orcid.org/0000-0002-7633-431X Jinyi Yang (1) https://orcid.org/0000-0001-5287-4242 Xiaohui Fan (ib https://orcid.org/0000-0003-3310-0131 Xue-Bing Wu (iD https://orcid.org/0000-0002-7350-6913 Minghao Yue (iD https://orcid.org/0000-0002-5367-8021 Jiang-Tao Li iD https://orcid.org/0000-0001-6239-3821 Fuyan Bian (iD https://orcid.org/0000-0002-1620-0897 Linhua Jiang (iD https://orcid.org/0000-0003-4176-6486 Eduardo Bañados (i) https://orcid.org/0000-0002-2931-7824 Jan-Torge Schindler (iD https://orcid.org/0000-00024544-8242

Joseph R. Findlay (iD https://orcid.org/0000-0002-3632-2015 Frederick B. Davies (i) https://orcid.org/0000-0003-0821-3644 Roberto Decarli (i) https://orcid.org/0000-0002-2662-8803 Emanuele P. Farina (i) https://orcid.org/0000-0002-6822-2254 Joseph F. Hennawi (1D https://orcid.org/0000-0002-7054-4332 Yun-Hsin Huang (iD https://orcid.org/0000-0003-4955-5632 Chiara Mazzuccheli (iD https://orcid.org/0000-0002-5941-5214 Ian D. McGreer (i) https://orcid.org/0000-0002-3461-5228 Bram Venemans (D) https://orcid.org/0000-0001-9024-8322 Fabian Walter (10) https://orcid.org/0000-0003-4793-7880 Simon Dye (i) https://orcid.org/0000-0002-1318-8343 Evan Haze Nunez (i) https://orcid.org/0000-0001-5595-757X

\section{References}

Allen, J. T., Hewett, P. C., Maddox, N., Richards, G. T., \& Belokurov, V. 2011, MNRAS, 410, 860

Arnaboldi, M., Neeser, M. J., Parker, L. C., et al. 2007, Msngr, 127, 28 Bañados, E., Venemans, B. P., Decarli, R., et al. 2016, ApJS, 227, 11

Bañados, E., Venemans, B. P., Mazzucchelli, C., et al. 2018, Natur, 553, 473 Bañados, E., Venemans, B. P., Morganson, E., et al. 2015, ApJ, 804, 118 Becker, G. D., \& Bolton, J. S. 2013, MNRAS, 436, 1023

Becker, R. H., White, R. L., \& Helfand, D. J. 1995, ApJ, 450, 559

Beckwith, S. V. W., Stiavelli, M., Koekemoer, A. M., et al. 2006, AJ, 132, 1729

Best, W. M. J., Liu, M. C., Magnier, E. A., et al. 2015, ApJ, 814, 118 Bolton, J. S., Haehnelt, M. G., Warren, S. J., et al. 2011, MNRAS, 416, L70 Boroson, T. A., \& Green, R. F. 1992, ApJS, 80, 109

Bosman, S. E. I., \& Becker, G. D. 2015, MNRAS, 452, 1105

Byard, P. L., \& O'Brien, T. P. 2000, Proc. SPIE, 4008, 934

Carilli, C. L., \& Walter, F. 2013, ARA\&A, 51, 105

Carilli, C. L., Wang, R., van Hoven, M. B., et al. 2007, AJ, 133, 2841

Casali, M., Adamson, A., Alves de Oliveira, C., et al. 2007, A\&A, 467, 777

Chambers, K. C., Magnier, E. A., Metcalfe, N., et al. 2016, arXiv:1612.05560 Condon, J. J., Cotton, W. D., Greisen, E. W., et al. 1998, AJ, 115, 1693

D’Aloisio, A., McQuinn, M., Davies, F. B., \& Furlanetto, S. R. 2018, MNRAS, 473,560

Davies, F. B., Hennawi, J. F., Bañados, E., et al. 2018a, ApJ, 864, 142

Davies, F. B., Hennawi, J. F., Eilers, A.-C., \& Lukić, Z. 2018b, ApJ, 855, 106 Decarli, R., Walter, F., Venemans, B. P., et al. 2017, Natur, 545, 457 Decarli, R., Walter, F., Venemans, B. P., et al. 2018, ApJ, 854, 97 Dey, A., Schlegel, D. J., Lang, D., et al. 2019, AJ, 157, 168

Dye, S., Lawrence, A., Read, M. A., et al. 2018, MNRAS, 473, 5113 Faber, S. M., Phillips, A. C., Kibrick, R. I., et al. 2003, Proc. SPIE, 4841, 1657 Fabricant, D. G., Fata, R. G., \& Epps, H. W. 1998, Proc. SPIE, 3355, 232 Fan, X., Carilli, C. L., \& Keating, B. 2006, ARA\&A, 44, 415 Fan, X., Narayanan, V. K., Lupton, R. H., et al. 2001, AJ, 122, 2833 Fan, X., Wang, F., \& Yang, J. 2019, ApJL, 870, L11 Findlay, J. R., Kobulnicky, H. A., Weger, J. S., et al. 2016, PASP, 128, 115003 Gibson, R. R., Jiang, L., Brandt, W. N., et al. 2009, ApJ, 692, 758 Górski, K. M., Hivon, E., Banday, A. J., et al. 2005, ApJ, 622, 759 Greig, B., Mesinger, A., Haiman, Z., \& Simcoe, R. A. 2017, MNRAS, 466, 4239

Haardt, F., \& Madau, P. 2012, ApJ, 746, 125

Hewett, P. C., \& Foltz, C. B. 2003, AJ, 125, 1784

Hook, I. M., Jørgensen, I., Allington-Smith, J. R., et al. 2004, PASP, 116, 425 Hopkins, P. F., Hernquist, L., Cox, T. J., et al. 2005, ApJ, 630, 705
Illingworth, G. D., Magee, D., Oesch, P. A., et al. 2013, ApJS, 209, 6 Irwin, M. J., Lewis, J., Hodgkin, S., et al. 2004, Proc. SPIE, 5493, 411 Jiang, L., Fan, X., Ivezić, Ž., et al. 2007, ApJ, 656, 680 Jiang, L., McGreer, I. D., Fan, X., et al. 2016, ApJ, 833, 222

Kashikawa, N., Ishizaki, Y., Willott, C. J., et al. 2015, ApJ, 798, 28 Kaurov, A. A., \& Gnedin, N. Y. 2015, ApJ, 810, 154

Kellermann, K. I., Sramek, R., Schmidt, M., Shaffer, D. B., \& Green, R. 1989, AJ, 98, 1195

Khaire, V., \& Srianand, R. 2015, MNRAS, 451, L30

Kirkpatrick, J. D., Cushing, M. C., Gelino, C. R., et al. 2011, ApJS, 197, 19

Knigge, C., Scaringi, S., Goad, M. R., \& Cottis, C. E. 2008, MNRAS, 386,1426

Koptelova, E., Hwang, C.-Y., Yu, P.-C., Chen, W.-P., \& Guo, J.-K. 2017, NatSR, 7, 41617

Kulkarni, G., Worseck, G., \& Hennawi, J. F. 2019, MNRAS, 488, 1035 Lang, D. 2014, AJ, 147, 108

Lawrence, A., Warren, S. J., Almaini, O., et al. 2007, MNRAS, 379, 1599

Lusso, E., Worseck, G., Hennawi, J. F., et al. 2015, MNRAS, 449, 4204

Madau, P., \& Haardt, F. 2015, ApJL, 813, L8

Madau, P., Haardt, F., \& Rees, M. J. 1999, ApJ, 514, 648

Madau, P., \& Rees, M. J. 2000, ApJL, 542, L69

Mainzer, A., Bauer, J., Grav, T., et al. 2011, ApJ, 731, 53

Mao, J., \& Kim, M. 2016, ApJ, 828, 96

Matsuoka, Y., Iwasawa, K., Onoue, M., et al. 2018a, ApJS, 237, 5

Matsuoka, Y., Onoue, M., Kashikawa, N., et al. 2016, ApJ, 828, 26

Matsuoka, Y., Onoue, M., Kashikawa, N., et al. 2018b, PASJ, 70, S35

Matsuoka, Y., Strauss, M. A., Kashikawa, N., et al. 2018c, ApJ, 869, 150

Mazzucchelli, C., Bañados, E., Venemans, B. P., et al. 2017, ApJ, 849, 91

McGreer, I. D., Fan, X., Jiang, L., \& Cai, Z. 2018, AJ, 155, 131

McGreer, I. D., Hall, P. B., Fan, X., et al. 2010, AJ, 140, 370

McGreer, I. D., Jiang, L., Fan, X., et al. 2013, ApJ, 768, 105

McLeod, B., Fabricant, D., Nystrom, G., et al. 2012, PASP, 124, 1318

McMahon, R. G., Banerji, M., Gonzalez, E., et al. 2013, Msngr, 154, 35

McQuinn, M., Oh, S. P., \& Faucher-Giguère, C.-A. 2011, ApJ, 743, 82

Meiksin, A. 2005, MNRAS, 356, 596

Meisner, A. M., Lang, D., \& Schlegel, D. J. 2017, AJ, 154, 161

Miralda-Escudé, J. 1998, ApJ, 501, 15

Mortlock, D. J., Warren, S. J., Venemans, B. P., et al. 2011, Natur, 474, 616

Oke, J. B., \& Gunn, J. E. 1982, PASP, 94, 586

Page, M. J., \& Carrera, F. J. 2000, MNRAS, 311, 433

Pentericci, L., Vanzella, E., Fontana, A., et al. 2014, ApJ, 793, 113

Planck Collaboration, Aghanim, N., Akrami, Y., et al. 2018, arXiv:1807.06209

Pons, E., McMahon, R. G., Simcoe, R. A., et al. 2019, MNRAS, 484, 5142

Reed, S. L., McMahon, R. G., Martini, P., et al. 2017, MNRAS, 468, 4702

Reichard, T. A., Richards, G. T., Hall, P. B., et al. 2003, AJ, 126, 2594

Richards, G. T., Strauss, M. A., Fan, X., et al. 2006, AJ, 131, 2766

Risaliti, G., \& Elvis, M. 2010, A\&A, 516, A89

Robertson, B. E., Ellis, R. S., Furlanetto, S. R., \& Dunlop, J. S. 2015, ApJL, 802, L19

Ross, N. P., Myers, A. D., Sheldon, E. S., et al. 2012, ApJS, 199, 3

Schindler, J.-T., Fan, X., McGreer, I. D., et al. 2018, ApJ, 863, 144

Schlegel, D. J., Finkbeiner, D. P., \& Davis, M. 1998, ApJ, 500, 525

Schmidt, G. D., Weymann, R. J., \& Foltz, C. B. 1989, PASP, 101, 713

Selsing, J., Fynbo, J. P. U., Christensen, L., \& Krogager, J.-K. 2016, A\&A, 585, A87

Semelin, B. 2016, MNRAS, 455, 962

Shankar, F., Crocce, M., Miralda-Escudé, J., Fosalba, P., \& Weinberg, D. H. 2010, ApJ, 718, 231

Shen, Y., Strauss, M. A., Oguri, M., et al. 2007, AJ, 133, 2222

Simcoe, R. A., Burgasser, A. J., Bernstein, R. A., et al. 2008, Proc. SPIE, 7014, $70140 \mathrm{U}$

Stevenson, K. B., Bean, J. L., Seifahrt, A., et al. 2016, ApJ, 817, 141

Stocke, J. T., Morris, S. L., Weymann, R. J., \& Foltz, C. B. 1992, ApJ, 396, 487

Tang, J.-J., Goto, T., Ohyama, Y., et al. 2017, MNRAS, 466, 4568

Tsuzuki, Y., Kawara, K., Yoshii, Y., et al. 2006, ApJ, 650, 57

van Leeuwen, F., Evans, D. W., De Angeli, F., et al. 2017, A\&A, 599, A32

Vanden Berk, D. E., Richards, G. T., Bauer, A., et al. 2001, AJ, 122, 549

Venemans, B. P., Bañados, E., Decarli, R., et al. 2015, ApJL, 801, L11

Venemans, B. P., Findlay, J. R., Sutherland, W. J., et al. 2013, ApJ, 779, 24

Venemans, B. P., Walter, F., Decarli, R., et al. 2017, ApJL, 851, L8

Vestergaard, M., \& Wilkes, B. J. 2001, ApJS, 134, 1

Volonteri, M., \& Rees, M. J. 2006, ApJ, 650, 669

Volonteri, M., Silk, J., \& Dubus, G. 2015, ApJ, 804, 148

Wang, F., Fan, X., Yang, J., et al. 2017, ApJ, 839, 27

Wang, F., Wu, X.-B., Fan, X., et al. 2015, ApJL, 807, L9 
Wang, F., Wu, X.-B., Fan, X., et al. 2016, ApJ, 819, 24

Wang, F., Yang, J., Fan, X., et al. 2018, ApJL, 869, L9

Wang, R., Carilli, C. L., Beelen, A., et al. 2007, AJ, 134, 617

Willott, C. J., Delorme, P., Reylé, C., et al. 2010, AJ, 139, 906

Wright, E. L., Eisenhardt, P. R. M., Mainzer, A. K., et al. 2010, AJ, 140, 1868

Wu, X.-B., Wang, F., Fan, X., et al. 2015, Natur, 518, 512
Wyithe, J. S. B., \& Loeb, A. 2003, ApJ, 595, 614

Yang, J., Wang, F., Fan, X., et al. 2019, ApJ, 871, 199

Yang, J., Wang, F., Wu, X.-B., et al. 2016, ApJ, 829, 33

York, D. G., Adelman, J., Anderson, J. E., Jr., et al. 2000, AJ, 120, 1579

Yuan, H., Zhang, H., Zhang, Y., et al. 2013, A\&C, 3, 65

Zou, H., Zhang, T., Zhou, Z., et al. 2018, ApJS, 237, 37 